International Journal of Bifurcation and Chaos, Vol. 17, No. 8 (2007) 2605-2623

(C) World Scientific Publishing Company

\title{
THE QUASI-PERIODIC REVERSIBLE HOPF BIFURCATION
}

\author{
HENK W. BROER \\ Instituut voor Wiskunde en Informatica, \\ Rijksuniversiteit Groningen, \\ Blauwborgje 3, 9747 AC Groningen, The Netherlands \\ M. CRISTINA CIOCCI \\ Department of Mathematics, Imperial College London, \\ Campus South Kensington, London, SW7 2AZ, UK \\ HEINZ HANßMANN \\ Mathematisch Instituut, Universiteit Utrecht, \\ Postbus 80.010, 3508 TA Utrecht, The Netherlands
}

Received February 28, 2006; Revised July 7, 2006

\begin{abstract}
We consider the perturbed quasi-periodic dynamics of a family of reversible systems with normally 1:1 resonant invariant tori. We focus on the generic quasi-periodic reversible Hopf bifurcation and address the persistence problem for integrable quasi-periodic tori near the bifurcation point. Using KAM theory, we describe how the resulting invariant tori of maximal and lower dimensions are parameterized by Cantor sets.
\end{abstract}

Keywords: KAM theory; versal unfolding; relative equilibria; 1:1 resonance.

\section{Introduction}

We consider reversible systems near a quasi-periodic invariant $n$-torus that is in a normal 1:1 resonance. Aim is to describe the dynamics of a generic unfolding model. It turns out that a quasi-periodic reversible Hopf bifurcation is involved. In a companion paper [Broer et al., 2006; Ciocci, 2003] persistence of (Diophantine) $n$-tori is established and their normal linear behavior is analyzed; in the present paper we focus on nonlinear aspects. Our approach merges reversible KAM theory with a theory of reversible normal forms. It is to be noted that a very similar scenario holds for the Hamiltonian case, cf. [Broer et al., 2007a; Broer et al., 2007b; Hoo, 2005], where a generic unfolding of the 1:-1 resonance $^{1}$ involves a quasi-periodic Hamiltonian
Hopf bifurcation; we largely follow the same strategy in the present reversible case. As we shall see below, the analogy of the reversible and Hamiltonian case also stretches out far into the mathematical contents.

Remark. Systems as discussed in this paper turn out to be relevant for hydrodynamic stability problems, cf. [Iooss et al., 1989; Iooss \& Los, 1990]. Indeed, by a center manifold argument, see [Mielke, 1986; Iooss et al., 1989], the following is proven. When the Navier-Stokes equations in a cylindrical domain are written as an evolution equation in an (unbounded) space variable, all (steady) solutions that stay small in amplitude are determined by the solutions of a four-dimensional reversible system

\footnotetext{
${ }^{1}$ Note that in the reversible case there is no difference between the $1: 1$ and $1:-1$ resonance.
} 
with a 1:1 resonance in the linear part, see [Iooss et al., 1989], p. 242.

A (small) quasi-periodic forcing then puts us into the context of the quasi-periodic reversible Hopf bifurcation. We expect that systems displaying a reversible Hopf bifurcation of equilibria (or periodic orbits) often have additional pairs of purely imaginary eigenvalues, whence excitation of the corresponding normal modes provides a possible mechanism within the system itself.

\section{Background}

Throughout, we work with the phase space $M=$ $\mathbb{T}^{n} \times \mathbb{R}^{4}=\{x, z\}$, where $\mathbb{T}^{n}=\mathbb{R}^{n} /\left(2 \pi \mathbb{Z}^{n}\right)$ is the $n$-dimensional torus with coordinates $x=\left(x_{1}, \ldots\right.$, $\left.x_{n}\right)(\bmod 2 \pi)$ and where $\mathbb{R}^{4}$ has coordinates $z=$ $\left(z_{1}, \ldots, z_{4}\right)$.

Given is an involution $G: M \rightarrow M$, of the form $G(x, z)=(-x, R z)$, where $R \in G l(4, \mathbb{R})$ is a linear involution, i.e. with $R^{2}=\mathrm{Id}$. This means that $R$ has only \pm 1 as eigenvalues. We assume that the eigenvalue 1 occurs with multiplicity 2 , which means that the fixed point space of $R$ has dimension 2 and, therefore, $G$ has a two-dimensional submanifold of fixed points.

We consider vector fields $X$ on $M$ of class $C^{\omega}$ (i.e. real analytic), that are reversible with respect to $G$, i.e. such that

$$
G_{*}(X)=-X
$$

Writing

$$
X(x, z)=\sum_{j=1}^{n} f_{j}(x, z) \frac{\partial}{\partial x_{j}}+\sum_{\ell=1}^{4} h_{\ell}(x, z) \frac{\partial}{\partial z_{\ell}},
$$

or, in shorthand notation $X=f \partial / \partial x+h \partial / \partial z$, then (1) rewrites to

$$
\begin{aligned}
& f(-x, R z) \equiv f(x, z) \\
& h(-x, R z) \equiv-R h(x, z) .
\end{aligned}
$$

Note that this reversibility means that $G$ takes integral curves of $X$ to integral curves of $X$, reversing the time-direction. The vector field $X$ is integrable if it is invariant under the natural $\mathbb{T}^{n}$-action

$$
\begin{aligned}
\left(x_{0},(x, z)\right) & \in \mathbb{T}^{n} \times\left(\mathbb{T}^{n} \times \mathbb{R}^{4}\right) \\
& \mapsto\left(x+x_{0}, z\right) \in \mathbb{T}^{n} \times \mathbb{R}^{4},
\end{aligned}
$$

which implies that the functions $f$ and $h$ do not depend on the $x$-variables.

Our interest is with invariant $n$-tori of the form $\mathbb{T}^{n} \times\left\{z_{0}\right\}$ of the integrable vector field $X$. Note that these tori are orbits of the natural $n$-torus action. These correspond to zeroes $z_{0}$ of the function $h$. We discuss persistence of such an $n$-torus under (not necessarily integrable) small perturbation, for simplicity putting $z_{0}=0$.

It turns out that we shall need the system to depend on parameters. Therefore we consider real analytic families $X=X(x, z, \nu)$, where $\nu$ runs over a $q$-dimensional parameter domain with $q \geq n+2$. In particular the coefficient functions of an integrable vector field become $f=f(z, \nu)$ and $h=$ $h(z, \nu)$.

Remark. A general treatment of local bifurcations in reversible systems is given in e.g. [Lamb, 1994]. Specifically, when the eigenvalues (Floquet multipliers) are in 1:1 resonance, a reversible Hopf bifurcation occurs which has characteristics very close to the Hamiltonian Hopf bifurcation, cf. [van der Meer et al., 1994; Bridges, 1998].

In this setting the main issue of KAM theory is the persistence of $X$-invariant $n$-tori under a nonintegrable perturbation. Starting point of our considerations is the normal linear (leading) part

$$
X^{N}(x, z, \nu)=\omega(\nu) \frac{\partial}{\partial x}+\Omega(\nu) \frac{\partial}{\partial z}
$$

of the family $X$ at the torus $z=0$. Compare with [Broer et al., 1990; Broer \& Huitema, 1995]. Here $\omega(\nu)=f(0, \nu)$ and $\Omega(\nu)=d_{z} h(0, \nu)$. From (1) we infer $\Omega \circ R=-R \circ \Omega$, i.e. the matrix $\Omega$ is infinitesimally reversible. The space of all such matrices is denoted by $g l_{-R}(4, \mathbb{R})$.

Remark. Hyperbolicity and ellipticity occur for open subsets of $g l_{-R}(4, \mathbb{R})$, where $\Omega$ is semi-simple. Most of the research thus far is devoted to KAM theory in this context, compare with [Moser, 1973; Sevryuk, 1986; Huitema, 1988; Broer et al., 1990; Broer \& Huitema, 1995; Wei, 2001], for more references see [Broer et al., 1996].

We assume Diophantine conditions on the internal and normal frequencies (related to the imaginary part of pairs or quadruples of complex eigenvalues), again see [Moser, 1973; Broer et al., 1990; Broer \& Huitema, 1995] and the other above references. Note that we need at least that the internal frequency vector $\omega=\omega(\nu)$ for certain positive constants $\gamma$ and $\tau$ satisfies

$$
|\langle\omega, k\rangle| \geq \gamma|k|^{-\tau}
$$


for all $k \in \mathbb{Z}^{n} \backslash\{0\}$. We recall the general fact, cf. [Broer et al., 1996], that for $\tau>n-1$ the subset of all such Diophantine $\omega$ in $\mathbb{R}^{n}$ is nowhere dense, but has positive Lebesgue measure, the complement of which gets small measure as $\gamma \downarrow 0$.

The family $X$ of vector fields is assumed to satisfy the Broer-Huitema-Takens (BHT) nondegeneracy condition at the invariant $n$-torus $z=0, \nu=\nu_{0}$. This nondegeneracy condition from [Huitema, 1988; Broer et al., 1990] is expressed in terms of the product map

$$
\nu \in \mathbb{R}^{q} \mapsto(\omega(\nu), \Omega(\nu)) \in \mathbb{R}^{n} \times g l_{-R}(4, \mathbb{R})
$$

derived from the normal linearization (4) of $X$.

The first factor is used to pull back the Cantorlike structure defined by Diophantine conditions from frequency space to parameter space; this can be fully achieved if $\nu \mapsto \omega(\nu)$ is submersive (i.e. has a surjective derivative at $\nu=\nu_{0}$ ). Similarly, we want to use the second factor to pull back a versal unfolding as obtained from the theory of unfolding of matrices (e.g. [Arnold, 1971; Gibson, 1979]). In the present reversible context this amounts to computing the codimension $c$ of the orbit of $\Omega\left(\nu_{0}\right)$ under the adjoint action $\operatorname{Ad}(A)(\Omega)=A \Omega A^{-1}$ of the group $G L_{R}(4, \mathbb{R})$ of $R$-equivariant matrices on $g l_{-R}(4, \mathbb{R})$ and to then consider a map $p: g l_{-R}(4, \mathbb{R}) \rightarrow \mathbb{R}^{c}$ where $\mathbb{R}^{c}$ parameterizes a section transverse to that $\operatorname{Ad}\left(G L_{R}(4, \mathbb{R})\right)$-orbit.

These two requirements are simultaneously achieved if $\nu \mapsto(\omega(\nu), p(\Omega(\nu)))$ is submersive. In addition to this, BHT-nondegeneracy requires $\operatorname{det} \Omega\left(\nu_{0}\right)$ to be non-zero. This approach surely needs a lot of parameters, but this number can be relaxed in various ways, compare with [Broer et al., 1990, part I, Ch. 7] or with [Broer et al., 1996].

The results in [Huitema, 1988; Broer \& Huitema, 1995] address the case that $\Omega$ has only simple eigenvalues; then $c=N_{1}+N_{2}+N_{3}$ where $2 N_{1}$ is the number of real eigenvalues (which form pairs $\alpha,-\alpha$ in the present reversible context), $N_{2}$ is the number of pairs of purely imaginary eigenvalues and $4 N_{1}$ is the number of remaining eigenvalues $\pm \alpha \pm \mathrm{i} \beta$. For $N_{2}=0$ the Diophantine conditions (5) suffice, but in the case $N_{2} \neq 0$ of additional normal frequencies these have to satisfy Diophantine conditions as in (18) below. Here the mapping $p$ can be chosen to map into the spectrum, as an unfolding is versal if all eigenvalues move. The lower dimensional KAM theory in [Huitema, 1988; Broer \& Huitema, 1995] roughly states that near a nondegenerate $n$ torus many $n$-tori persist under small perturbation.
For nonsimple eigenvalues $\Omega\left(\nu_{0}\right)$ is not in one of the elliptic or hyperbolic codimension 0 strata inside $g l_{-R}(4, \mathbb{R})$ and the numbers $N_{1}, N_{2}, N_{3}$ may change under parameter variation whence we speak of a bifurcation.

\section{The Perturbation Problem}

Presently our interest is with the 1:1 resonance where $\Omega\left(\nu_{0}\right)$ has a double pair of eigenvalues on the imaginary axis, with algebraic multiplicity 2 . We restrict to the generic subcase of geometric multiplicity 1 , where $\Omega\left(\nu_{0}\right)$ has a non-zero nilpotent part. For $\Omega\left(\nu_{0}\right) \in g l_{-R}(4, \mathbb{R})$ BHT-nondegeneracy amounts to (trans)-versality of the unfolding $\Omega(\nu)$ to the orbit of $\Omega\left(\nu_{0}\right)$ under the natural action of the $R$-equivariant linear group $G l_{R}(4, \mathbb{R})$, compare with [Arnold, 1971; Broer \& Huitema, 1995; Ciocci, 2003; Broer et al., 2006]. For the eigenvalue configurations of $\Omega(\nu)$ see Fig. 1.

Let us now describe the set up in more detail. Starting point is an integrable family $X=X_{\nu}(x, z)$ as described in the introduction, defined on the phase space $\mathbb{T}^{n} \times \mathbb{R}^{4}=\{x, z\}$. The normal linear part has the form (4). Dividing out the $\mathbb{T}^{n}$ symmetry leads to the reduced family

$$
X_{\text {red }}(z, \nu)=(\Omega(\nu)+\text { h.o.t. }) \frac{\partial}{\partial z}
$$

of $R$-reversible vector fields on $\mathbb{R}^{4}=\{z\}$. Recall that $\Omega_{0}:=\Omega(0)$ is in $1: 1$ resonance. We first specify the reversor $R$ and the matrix $\Omega_{0}$, and then choose an appropriate versal unfolding $\nu \mapsto \Omega(\nu)$ in $g l_{-R}(4, \mathbb{R})$, compare with [Broer et al., 2006; Ciocci, 2003; Vanderbauwhede, 1990].

Proposition 1. Assume that $\Omega_{0} \in g l_{-R}(4, \mathbb{R})$ has eigenvalues $\pm \mathrm{i}$ with algebraic multiplicity 2 , and that $R \in G L(4, \mathbb{R})$ is a linear involution such that $\operatorname{dim}(\operatorname{Fix}(R))=2$. Then, there exists a basis of $\mathbb{R}^{4}$
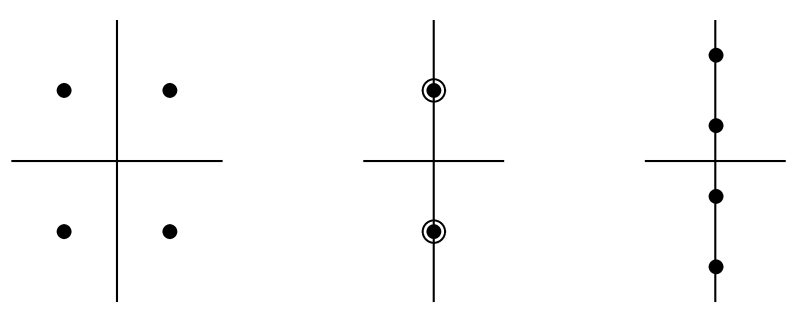

Hyperbolic: $\mu_{2}<0$

Doubly elliptic: $\mu_{2}=0$

Elliptic: $\mu_{2}>0$

Fig. 1. Position of the eigenvalues in a generic 1:1 resonance. A dot denotes a single eigenvalue, a circle-dot a double eigenvalue. 
with respect to which $R$ has the form

$$
R=\left(\begin{array}{cc}
R_{0} & O \\
O & -R_{0}
\end{array}\right), \quad \text { with } R_{0}:=\left(\begin{array}{rr}
1 & 0 \\
0 & -1
\end{array}\right)
$$

and in the case when $\Omega_{0}$ is not semisimple

$$
\Omega_{0}=S_{0}+N_{0}:=\left(\begin{array}{cc}
J & O \\
O & J
\end{array}\right)+\left(\begin{array}{cc}
O & -\mathrm{I} \\
O & O
\end{array}\right),
$$

where

$$
J:=\left(\begin{array}{rr}
0 & 1 \\
-1 & 0
\end{array}\right)
$$

Proof. See e.g. [Ciocci, 2003].

We refer to $S_{0}$ as to the semisimple part of $\Omega_{0}$, and to $N_{0}$ as its nilpotent part, cf. [Hirsch \& Smale, 1974]. The universal unfolding of $\Omega_{0}$ is constructed as the linear centralizer unfolding $\Omega(\nu)=\Omega_{0}+A(\mu)$ with appropriately chosen

$$
\begin{aligned}
A(\mu) \in C_{-}\left(\Omega_{0}\right):= & \operatorname{ker}\left(\operatorname{ad}\left(S_{0}\right)\right) \cap \operatorname{ker}\left(\operatorname{ad}\left(N_{0}^{T}\right)\right) \\
& \cap g l_{-R}(4, \mathbb{R}) .
\end{aligned}
$$

For more details see [Ciocci, 2003; Broer et al., 2006], where this result is more generally derived for $\Omega_{0} \in g l_{-R}(2 p, \mathbb{R})$, also compare with [van der Meer et al., 1994]. In the case of $\Omega_{0}$ given by (7), a direct computation reveals

$$
\Omega(\mu)=\left(\begin{array}{cc}
J & -\mathrm{I} \\
0 & J
\end{array}\right)+\left(\begin{array}{cc}
\mu_{1} J & O \\
\mu_{2} \mathrm{I} & \mu_{1} J
\end{array}\right)
$$

with $\left(\mu_{1}, \mu_{2}\right) \in \mathbb{R}^{2}$ varying over a neighborhood of 0 . Observe that $\Omega(\mu)-\Omega_{0}$ depends linearly on $\mu \in$ $\mathbb{R}^{2}$. From now on we assume $\Omega(\mu)$ of the form (8). In the reduced setting one can reparameterize $\nu$ to coincide with $\mu$, but in the nearly integrable setting we shall need to cover the frequencies $\omega$ as well, writing $\nu=(\omega, \mu)$.

In the sequel the eigenvalues of $\Omega(\mu)$ play an important role. From (8) we determine the four eigenvalues as

$$
\pm \mathrm{i}\left(1+\mu_{1}\right) \pm \sqrt{-\mu_{2}} .
$$

As $\mu_{2}$ crosses zero from negative to positive, a hyperbolic quadruple of eigenvalues turns into two pairs of purely imaginary eigenvalues, thus stabilizing the origin of $\mathbb{R}^{4}$ as an equilibrium of $X_{\text {red }}$, compare with Fig. 1. Observe that equilibria of $X_{\text {red }}$ correspond to relative equilibria of the full system. We denote by $\omega^{N}(\mu)$ the normal frequency vector consisting of the positive imaginary parts of the eigenvalues of $\Omega(\mu)$, i.e.

$$
\omega^{N}(\mu)=\left\{\begin{array}{llc}
\left(1+\mu_{1}, 1+\mu_{1}\right) & & \mu_{2} \leq 0 \\
\left(1+\mu_{1}+\sqrt{\mu_{2}},\right. & \text { for } & \\
\left.1+\mu_{1}-\sqrt{\mu_{2}}\right) & & \mu_{2}>0
\end{array} .\right.
$$

A reversible normal form analysis as given in Appendices A.2 and A.3 reveals that the normalized vector field on $\mathbb{T}^{n} \times \mathbb{R}^{4}$ reads

$$
X(x, z, \nu)=\tilde{X}+\text { h.o.t. }
$$

where the integrable part $\tilde{X}$ is given by

$$
\tilde{X}=f(z, \nu) \frac{\partial}{\partial x}+\tilde{X}_{\text {red }}
$$

with $\omega(\nu)=f(0, \nu)$ and $f(R z, \nu)=f(z, \nu)$ by the reversibility. Also, a general third order part of the family $\tilde{X}_{\text {red }}$ reads

$$
\begin{aligned}
\dot{z}_{1}= & \left(1+\mu_{1}\right) z_{2}-z_{3}+(\alpha+\gamma) z_{2} M+\delta z_{2} S \\
\dot{z}_{2}= & -\left(1+\mu_{1}\right) z_{1}-z_{4}-(\alpha+\gamma) z_{1} M-\delta z_{1} S \\
\dot{z}_{3}= & \left(1+\mu_{1}\right) z_{4}+\mu_{2} z_{1}+\beta z_{1} M \\
& +(\alpha+\gamma) z_{4} M+\gamma z_{1} S+\delta z_{4} S \\
\dot{z}_{4}= & -\left(1+\mu_{1}\right) z_{3}+\mu_{2} z_{2}+\beta z_{2} M \\
& -(\alpha+\gamma) z_{3} M+\gamma z_{2} S-\delta z_{3} S .
\end{aligned}
$$

Here $\mu=\left(\mu_{1}, \mu_{2}\right)$ is an unfolding parameter as before, while $\alpha, \beta, \gamma$ and $\delta$ are real constants. Moreover,

$$
\begin{aligned}
M & :=\frac{1}{2}\left(z_{1}^{2}+z_{2}^{2}\right), \\
N & :=\frac{1}{2}\left(z_{3}^{2}+z_{4}^{2}\right), \\
S & :=z_{1} z_{4}-z_{2} z_{3} .
\end{aligned}
$$

Compare with [Bridges, 1998; Iooss et al., 1989].

The system $\tilde{X}_{\text {red }}$ yields equilibria as well as periodic, quasi-periodic, homoclinic and heteroclinic orbits, see e.g. [Iooss et al., 1989]. We are interested in the fate of the periodic and quasiperiodic orbits. Relevant is that the family $\tilde{X}_{\text {red }}$ undergoes a generic reversible Hopf bifurcation as $\mu_{2}$ passes through 0 .

The bifurcation scenarios are easily read off from Figs. 2 and 3, compare also with Fig. 4. In both the supercritical case $\beta>0$ and the subcritical case $\beta<0$ the local stratification near the resonance is given by parts of the swallowtail catastrophe set, cf. [van der Meer, 1985]. 


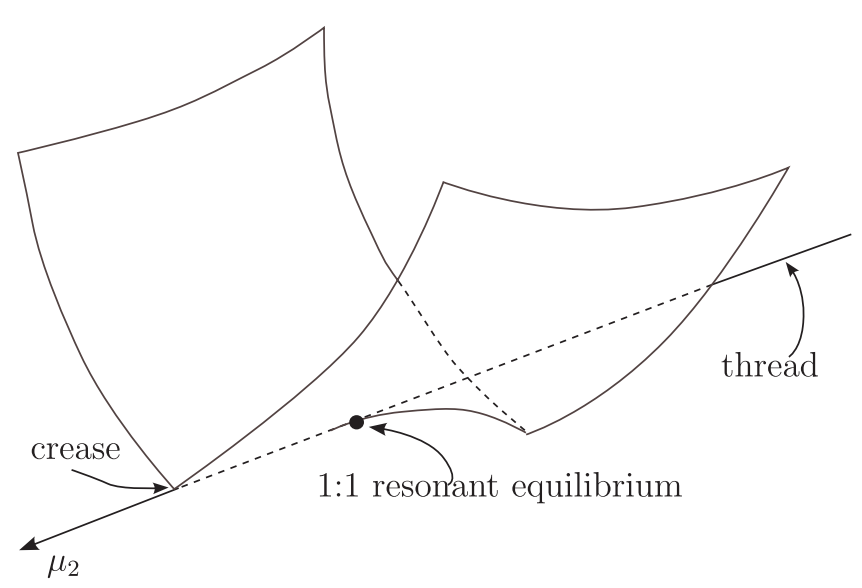

Fig. 2. Sketch of the local stratification near the 1:1 resonance. Supercritical case $(\beta>0)$ : the $\mu_{2}$-axis corresponds to the family of equilibria containing the resonant point. The surface parameterizing the (elliptic) periodic orbits consists of one sheet, forming a crease at $\mu_{2}>0$ where the equilibrium is elliptic, while the hyperbolic equilibria are parameterized by a thread that detaches from the sheet during the reversible Hopf bifurcation. The open domain above the sheet (in particular, surrounding the thread) parameterizes invariant 2-tori.

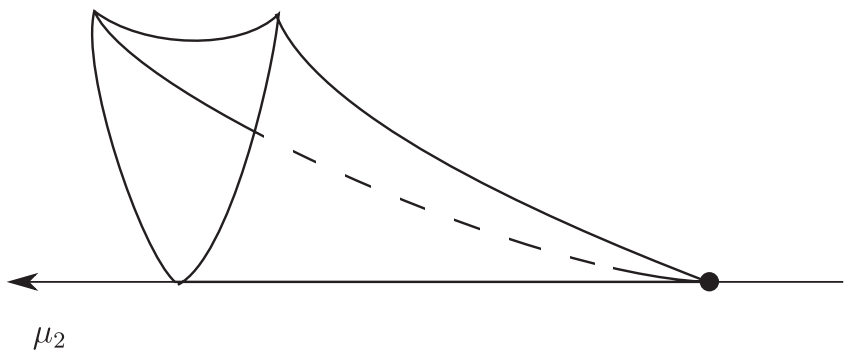

Fig. 3. Sketch of the local stratification near the 1:1 resonance. Subcritical case $(\beta<0)$ : the $\mu_{2}$-axis corresponds to the family of equilibria containing the resonant point. The surface parameterizing the periodic orbits consists of two sheets. The lower sheet parameterizes elliptic periodic orbits and forms a crease at the $\mu_{2}>0$ half axis where the equilibrium is elliptic. The upper sheet parameterizes hyperbolic periodic orbits and meets the lower sheet in a cusp-like fashion. Here a periodic center-saddle bifurcation takes place and correspondingly the two cusp lines parameterize parabolic periodic orbits. The open domain between the two sheets parameterizes invariant 2-tori. Outside that cone, in particular, for $\mu_{2}<0$, there are no 2-tori invariant under (13).

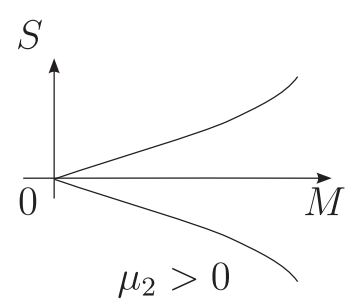

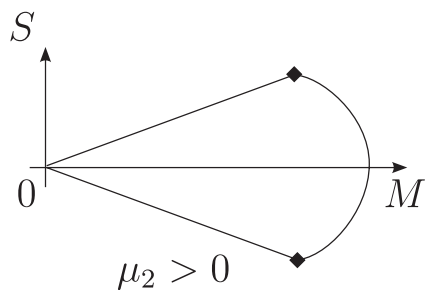

(b)

Fig. 4. Amplitude diagrams. (a) Supercritical case. Comparing with Fig. 2, the two lines emanating from the origin correspond to the sheet forming the crease and the curve detached from the origin is the part of the swallow tail passing below the thread. (b) Subcritical case. Comparing with Fig. 3 we have that the two lines emanating from the origin correspond to the sheet forming the crease, the arc between the diamonds represents the upper sheet of hyperbolic periodic solutions and the two diamonds stand for the curves of cusps where the two sheets meet, here the invariant periodic orbits are parabolic.

It is straightforward to reconstruct the dynamics of $\tilde{X}$ on $\mathbb{T}^{n} \times \mathbb{R}^{4}$ from the dynamics of $\tilde{X}_{\text {red }}$ on $\mathbb{R}^{4}$, see Sec. 4.2 below. Essentially, equilibria become invariant $n$-tori, periodic orbits turn into invariant $(n+1)$-tori and invariant 2 -tori of $\tilde{X}_{\text {red }}$ give rise to invariant $(n+2)$-tori of $\tilde{X}$.

KAM theory comes into play when addressing the question what remains of the above bifurcation scenario in the nearly integrable case; that is, what can be said about the full system $X$ as in (11). It turns out that a lot of invariant tori persist for nearly integrable perturbations. To establish this we first need linear stability of the $n$-torus family, which implies persistence for a suitable set of parameters. Secondly, applying quasi-periodic normal form theory, we can turn to the invariant $(n+1)$-tori, and then to the $(n+2)$-tori.

The frequency vectors of persistent tori typically satisfy Diophantine conditions, and we use in turn the Diophantine frequency vectors to parameterize persistent tori. This leads to so-called Cantor families of tori, to unions of invariant tori that locally are (Whitney)-smooth images of the product of a torus with a Cantor set.

\section{The Integrable Case}

The phase space of the integrable family $\tilde{X}$ is, near the normally 1:1 resonant $n$-torus $T_{0}^{n}=\mathbb{T}^{n} \times\{0\}$, foliated by invariant tori of dimensions $n, n+1$, 
$n+2$. The internal frequency vector of $\mathcal{T}_{0} \subset \mathbb{T}^{n} \times \mathbb{R}^{4}$ is denoted by $\omega$, and the normal frequencies are given by the positive imaginary parts of the eigenvalues of $\Omega(\mu)$ as in (10).

It turns out that the setting has many Hamiltonian characteristics, cf. [van der Meer et al., 1994; Lamb et al., 2001; Broer et al., 2007c; Hoo, 2005]. We here give precise statements, and for the proof refer to Appendix B.

Proposition 2. By means of an R-equivariant change of variables the normal form (12) of the vector field $X$ on $\mathbb{T}^{n} \times \mathbb{R}^{4}$ can be put into the form

$$
\tilde{X}=f \frac{\partial}{\partial x}+X_{H}+\alpha Y
$$

where $X_{H}$ is the Hamiltonian vector field with respect to the symplectic structure $\mathrm{d} z_{4} \wedge \mathrm{d} z_{2}+\mathrm{d} z_{3} \wedge$ $\mathrm{d} z_{1}$ with Hamiltonian

$$
\begin{aligned}
H(S, N, M)= & \omega^{N} S+N+\lambda M+\frac{\beta}{2} M^{2} \\
& +\gamma S M+\frac{\delta}{2} S^{2}+\text { h.o.t. }
\end{aligned}
$$

The vector field $Y$ is parallel to $X_{S}$ and vanishes at $\{M=0\}$.

Proof. See Appendix B.1.

We now sum up the main results giving the bifurcation scenario at the (nonsemisimple) 1:1 resonance for the four-dimensional reversible system $\tilde{X}_{\text {red }}$ and then give the corresponding results for the full integrable system $\tilde{X}$.

\subsection{Reduced integrable dynamics}

The following theorems summarize the results for the dynamics of $\tilde{X}_{\text {red }}$, for the proofs see Sec. 6.1. A sketch of the situation is given in Figs. 2 and 3. Note that these dynamics, with the quasiperiodic part reduced, match up with the results of [Vanderbauwhede, 1990; Knobloch \& Vanderbauwhede, 1995, 1996] for their simplest case $k=2$ after reduction of the periodic dynamics.

Theorem 3 (Supercritical reversible Hopf bifurcation). Given is the phase space $\mathbb{R}^{4}$ with the involution $R \in G L(4, \mathbb{R})$ as in $(6)$. Let $\tilde{X}_{\text {red }}$ be a family of $R$-reversible vector fields with a 1:1 resonance as before and with truncated normal form as in (13). Suppose $\beta>0$. (i) The point $z=0$ is an equilibrium for all values of the parameter $\mu$. It is hyperbolic for $\mu_{2}<0$ and elliptic for $\mu_{2}>0$. See Fig. 1.

(ii) In suitable coordinates, the periodic solutions of the system are given by the roots of the cubic equation

$$
\sigma^{2}-4 \beta M^{3}-4 \mu_{2} M^{2}-4 \gamma \sigma M^{2}=0,
$$

where $\sigma \neq 0$ is a fixed value of $S$ (14c) and $M$ is as in $(14 a)$. These are all elliptic. Also, for fixed $\mu_{2}>0$, they shrink down to the elliptic equilibrium.

(iii) The open part of parameter space above the surface defined by (17) (not on the "thread", $z=0)$ is filled with invariant 2-tori which are normally trivial.

Remark. $\tilde{X}_{\text {red }}$ as in (13) has the invariant functions $H, S: \mathbb{R}^{4} \rightarrow \mathbb{R}$ given by (14c) and (16). They define a ramified 2-torus bundle, whose singular fibers are described by (i) and (ii) of the theorem above. In particular, the regular part described by (iii) is not simply connected. We discuss below that this leads to monodromy.

Theorem 4 (Subcritical reversible Hopf bifurcation). Let $\tilde{X}_{\text {red }}$ be as in Theorem 3, except that $\beta<0$. Then,

(i) the point $z=0$ is an equilibrium for all values of the parameter $\mu$. It is hyperbolic for $\mu_{2}<0$ and elliptic for $\mu_{2}>0$.

(ii) There are no periodic orbits for $\mu_{2} \leq 0$. If $\mu_{2}>0$, the periodic solutions of the system are given in suitable coordinates by the roots of the cubic equation (17). The orbits are either elliptic or hyperbolic depending on which sheet we are of the surface, and they are parabolic on the cusp line $16 \mu_{2}^{3}=27 \beta^{2} \sigma^{2}$, see Fig. 3. All these orbits shrink down to the elliptic equilibrium as $\mu_{2} \searrow 0$.

(iii) The open part of parameter space bounded by the surface defined by (17) for $\mu_{2}>0$ is filled with invariant 2-tori which are normally trivial.

Remark. The bifurcation scenario of the reversible Hopf bifurcation is completely governed by the unfolding parameter $\mu_{2}$. The unfolding parameter $\mu_{1}$ detunes the normal frequency $\omega^{N}$ and becomes important only for the persistence of the full dynamics addressed in Sec. 5 . 


\subsection{Integrable dynamics}

We return to the setting of Sec. 3 and consider the real analytic family of integrable reversible vector fields $\tilde{X}(x, z ; \nu)$ given by $(12)$ on the phase space $\mathbb{T}^{n} \times \mathbb{R}^{4}$, with parameter $\nu=(\omega, \mu) \in \mathbb{R}^{n} \times \mathbb{R}^{2}$ varying over a small neighborhood of $\nu_{0}=\left(\omega_{0}, 0\right)$. This family has an invariant family of $n$-tori $\mathcal{T}^{n}=$ $\bigcup_{\nu} T_{\nu}^{n}$, with $T_{\nu}^{n}=\mathbb{T}^{n} \times\{z=0\} \times\{\nu\}$. By assumption the invariant $n$-torus $T_{0}=T_{\nu_{0}}^{n}$ is normally $1: 1$ resonant and the vector field $\tilde{X}_{\nu}$ is assumed to be nondegenerate at $T_{0}$. Roughly speaking the dynamics of $\tilde{X}_{\nu}$ in phase space looks like that of $\tilde{X}_{\text {red }}$ times $\mathbb{T}^{n}$, that is, near the normally resonant $T_{0}$ we have a ramified $(n+2)$-torus bundle, the singular fibres of which are invariant $n$-tori and $(n+1)$-tori, together with their stable and unstable manifolds. The corresponding stratification in the product of phase space and parameter space is described by parts of the swallow tail surface as sketched in Figs. 2 and 3.

Corollary 5 (Invariant $n$-tori). Let $\tilde{X}(x, z ; \nu)$ be a real analytic family of integrable reversible vector fields as in (12) on the phase space $\mathbb{T}^{n} \times \mathbb{R}^{4}$, with parameter $\nu=(\omega, \mu) \in \mathbb{R}^{n} \times \mathbb{R}^{2}$. The parallel $n$ torus $T_{\nu}^{n}$ is invariant under $\tilde{X}_{\nu}$ for all $\nu$ and it is normally hyperbolic for $\mu_{2}<0$, normally elliptic for $\mu_{2}>0$ and normally 1:1 resonant for $\mu_{2}=0$.

Proof. The integrable dynamics on $\mathbb{T}^{n} \times \mathbb{R}^{4}$ decouples into the parallel flow on the first factor and the flow of $\tilde{X}_{\text {red }}$ on the second factor.

Corollary 6 (Invariant $(n+1)$-tori). The integrable family $\tilde{X}(x, z ; \nu)$ above has a family of invariant $(n+1)$-tori, $T_{\nu, \sigma}^{n+1}$, determined by the cubic equation (17), where we use the value $\sigma$ of the $X_{H^{-}}$ invariant function $S$ as a distinguished parameter. There are two different cases:

(i) supercritical, $\beta>0$ : the invariant $(n+1)$-tori exist for all $\mu_{2}$. For $\mu_{2}>0$ they shrink down to $T_{\nu}^{n}$, while the family $T_{\nu, \sigma}^{n+1}$ is detached from $T_{\nu}^{n}$ for $\mu_{2}<0$. Moreover, all $(n+1)$-tori are all normally elliptic. See Fig. 2.

(ii) subcritical, $\beta<0$ : the invariant $(n+1)$-tori exist only for $\mu_{2}>0$. They can be classified into three groups according to their (normal) stability type: normally elliptic $T_{\nu, \sigma}^{n+1 ; \text { ell }}$, they are on the lower sheet of the swallowtail surface (17); normally hyperbolic $T_{\nu, \sigma}^{n+1 \text {;hyp }}$, they are on the upper sheet of (17); normally parabolic $T_{\nu, \sigma}^{n+1 ; p a r}$ on the border line where the two previous sheets from a cusp and meet. See Fig. 3.
Proof. Superposition of the conditionally periodic dynamics on the first factor of $\mathbb{T}^{n} \times \mathbb{R}^{4}$ with a periodic orbit on the second factor yields conditionally periodic dynamics with $n+1$ frequencies.

Recall that the elliptic and hyperbolic tori become parabolic when they meet and then vanish in a quasi-periodic center-saddle bifurcation, see e.g. [Hanßmann, 1998] for details on the similar scenario in the Hamiltonian case.

Corollary 7 (Invariant $(n+2)$-tori). Let $\tilde{X}(x$, $z ; \nu)$ be as before. Then in an open part of the product of phase space and parameter space there exists a family of invariant parallel $(n+2)$-tori, $T_{\nu, \sigma, \eta}^{n+2}$, which are normally trivial. Here $\eta$ is a distinguished parameter corresponding to the value of the function $H(16)$.

Proof. Superposition of the conditionally periodic dynamics on the first factor of $\mathbb{T}^{n} \times \mathbb{R}^{4}$ with conditionally periodic dynamics on the second factor yields conditionally periodic dynamics with $n+2$ frequencies.

Note that as in the corresponding Hamiltonian case, cf. e.g. [Broer et al., 2007c; Efstathiou, 2005; Hoo, 2005], the integrable bundle of $\tilde{X}$-invariant $(n+2)$-tori is nontrivial in the supercritical case $\beta>0$. Indeed, a (small) circle around the thread collects nontrivial monodromy. By the methods of Broer et al. [2007a] this nontriviality also holds for Whitney smooth interpolations of the nearlyintegrable and Cantorised bundle.

\section{KAM Theory}

As observed so far, the phase space of $\tilde{X}$ near the normally 1:1 resonant $n$-torus $T_{0}$ is foliated by tori of dimensions $n, n+1$ and $n+2$. In parameter space this foliation is described by parts of surface of the swallow tail catastrophe. The persistence of these invariant tori under small (nonintegrable) perturbations can partly be obtained by "standard" KAM theory and is partly addressed in [Broer et al., 2006]. With the assumption of nondegeneracy (see Sec. 2) for the vector fields $\tilde{X}$, by the versality of $\Omega$, the Inverse Function Theorem and a suitable reparameterization, cf. [Broer et al., 2006], the (nonintegrable) family $X$ has the form

$$
X_{\mu, \omega}=\tilde{X}+\text { h.o.t. }=\omega \frac{\partial}{\partial x}+\Omega(\mu) z \frac{\partial}{\partial z}+\Psi,
$$


with $\mu=\left(\mu_{1}(\nu), \mu_{2}(\nu)\right) \in \mathbb{R}^{2}$ such that $\left(\mu_{1}\left(\nu_{0}\right)\right.$, $\left.\mu_{2}\left(\nu_{0}\right)\right)=(0,0)$ and $\Omega(\mu)$ as in (8) and $\Psi$ containing the nonlinear terms.

\subsection{Persistence of $n$-tori}

As already observed the family $\tilde{X}$ has an invariant $n$-torus family: the union of $n$-tori $\mathcal{T}^{n}=\cup_{\nu} T_{\nu}^{n}$ parameterized by $\nu=(\omega, \mu)$, with $T_{\nu}^{n}=\mathbb{T}^{n} \times\{z=$ $0\} \times\{\nu\}$. The standard KAM theory (e.g. [Broer \& Huitema, 1995; Broer et al., 1996]) yields persistence for subfamilies of the family $\mathcal{T}_{\nu}^{n}$ containing elliptic and hyperbolic tori. By [Broer et al., 2006] we can handle the problem of multiple Floquet exponents and obtain the persistence of the family of $n$-tori containing the resonant 1:1 torus.

Loosely speaking, it turns out that in a nondegenerate family, many of the tori $\mathcal{T}_{\nu}^{n}$, for $\nu$ near $\nu_{0}$, survive nonintegrable perturbation, again given suitable Diophantine conditions, which now differ in the various regions of the parameter space. This is a direct consequence of [Broer et al., 2006] (see also [Ciocci, 2005, 2003]).

In the sequel we will denote by $\Gamma_{\tau, \gamma}(U)$ the set of Diophantine parameters given by

$$
\begin{aligned}
\Gamma_{\tau, \gamma}(U)= & \left\{(\omega, \mu) \in U||\langle\omega, k\rangle+\left\langle\omega^{N}(\mu), \ell\right\rangle \mid\right. \\
& \geq \gamma|k|^{-\tau}, \text { for all } k \in \mathbb{Z}^{n} \backslash\{0\} \text { and all } \\
& \left.\ell \in \mathbb{Z}^{2} \text { with }\left|\ell_{1}\right|+\left|\ell_{2}\right| \leq 2\right\} .
\end{aligned}
$$

The set $\Gamma_{\tau, \gamma}\left(U^{\prime}\right)$ is the subset where the set $U^{\prime} \subset U$ is given by

$$
U^{\prime}:=\left\{\nu \in U: \operatorname{dist}\left(\left(\omega(\nu), \omega^{N}(\nu)\right), \partial \mathcal{F}(U)\right)>\gamma\right\} .
$$

Here $\mathcal{F}: \mathbb{R}^{q} \rightarrow \mathbb{R}^{n} \times \mathbb{R}^{2}, \nu \mapsto\left(\omega(\nu), \omega^{N}(\nu)\right)$ is the frequency map with the normal frequency vector $\omega^{N}(\nu)$ given by $(10)$.
Geometry of Diophantine conditions. As said before $\nu=(\omega, \mu) \in \mathbb{R}^{n} \times \mathbb{R}^{2}$, where we take an open subset $U$ containing the resonant point $\mu_{2}=0$. Generally speaking, for $\tau>n-1$ and $\gamma>0$ we consider the set $\Gamma_{\tau, \gamma}(U)$ as in (18) with the normal frequency $\omega^{N}(\mu)$ as in (10). Note that for $\ell=0$, we just retrieve (5). Since $\omega^{N}(\mu)$ differs for $\mu_{2}<0$ and $\mu_{2}>0$, we split $U=U_{-} \cup U_{0} \cup U_{+}$according to the sign of $\mu_{2}$. The Diophantine conditions so give rise to

$$
\Gamma_{\tau, \gamma}(U)=\Gamma_{\tau, \gamma}\left(U_{-}\right) \cup \Gamma_{\tau, \gamma}\left(U_{0}\right) \cup \Gamma_{\tau, \gamma}\left(U_{+}\right) .
$$

From (10) we see that $\Gamma_{\tau, \gamma}\left(U_{+}\right)$is determined by the conditions

$$
\left|\langle\omega, k\rangle+l_{1}\left(1+\mu_{1}\right) \pm l_{2} \sqrt{\mu_{2}}\right| \geq \gamma|k|^{-\tau},
$$

for all $k \in \mathbb{Z}^{n} \backslash\{0\}$ and $l \in \mathbb{R}^{2}$ with $\left(l_{1}, l_{2}\right)=$ $(0,0),(1,1),(2,2),(2,0),(0,2)$. It follows that $\Gamma_{\tau, \gamma}\left(U_{+}\right)$is diffeomorphic to the union of half closed parabolæ,

$$
\left\{\left(r \omega, r \mu_{1}, r^{2} \mu_{2}\right) \in \mathbb{R}^{n} \times \mathbb{R}^{2} \mid r \geq 1\right\} .
$$

Similarly it follows from (10) that $\Gamma_{\tau, \gamma}\left(U_{-}\right)$is the union of half planes of the form

$$
\left\{\left(r \omega, r \mu_{1}, s \mu_{2}\right) \in \mathbb{R}^{n} \times \mathbb{R}^{2} \mid r, s \geq 1\right\} .
$$

See Fig. 5.

Theorem 8 (Persistence of $n$-tori [Broer et al., 2006]). Let $\tilde{X}_{\nu}$ be a family of real analytic reversible integrable vector fields on $\mathbb{T}^{n} \times \mathbb{R}^{4}$ as described in the introduction. Suppose that the family is nondegenerate at the invariant torus $T_{0}=\mathbb{T}^{n} \times\{z=$ $0\} \times\left\{\nu_{0}\right\}$ and that this torus is in generic normal 1:1 resonance. Let $X$ be a family of real analytic reversible vector fields close to $\tilde{X}$ in the

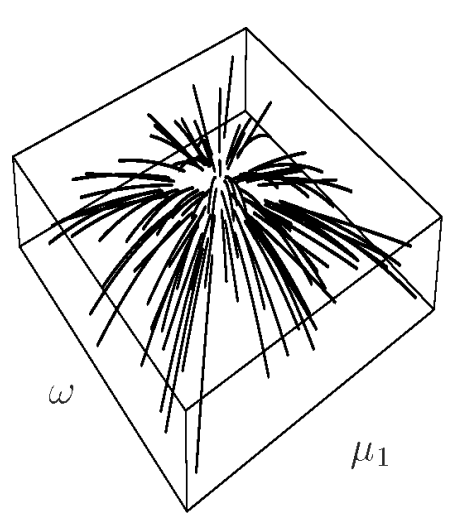

(a)

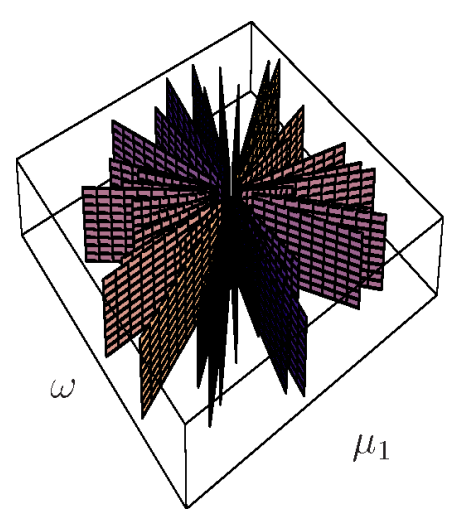

(b)

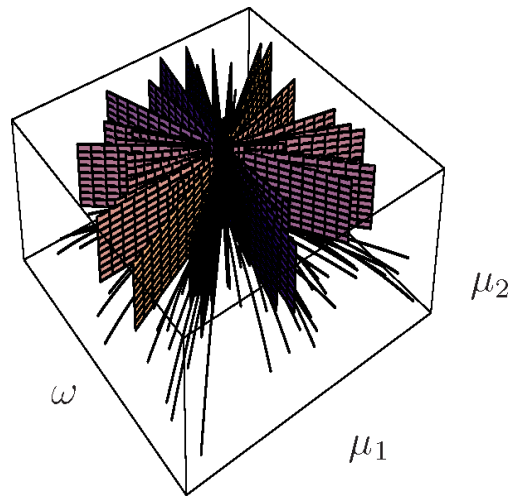

(c)

Fig. 5. (a, b): Sketch of "Cantor" sets in the $\left(\omega, \mu_{1}, \mu_{2}\right)$-space obtained by intersecting $\Gamma_{\tau, \gamma}\left(U_{+}\right)$and $\Gamma_{\tau, \gamma}\left(U_{-}\right)$with $\omega_{0} \mathbb{R}$ for a fixed Diophantine $\omega_{0}$. (c) Similar sketch for $\Gamma_{\tau, \gamma}(U)$ by matching the contributions for $U_{+}$and $U_{-}$. 
compact open topology on complex analytic extensions. Then, for $\gamma>0$ sufficiently small and $\tau>$ $n-1$, there exist a neighborhood $U$ of $\nu_{0} \in \mathbb{R}^{q}$ and a map $\Phi: M \times U \rightarrow M \times \mathbb{R}^{q}$, defined near $T_{\nu_{0}}$, such that

(i) $\Phi$ is a $C^{\infty}$-near-identity diffeomorphism onto its image;

(ii) the image of $V=\mathbb{T}^{n} \times\{z=0\} \times \Gamma_{\tau, \gamma}\left(U^{\prime}\right)$ under $\Phi$ is a Cantor family of $X$-invariant Diophantine tori and the restriction of $\left.\Phi\right|_{V}$ is a conjugacy between $\tilde{X}$ and $X$;

(iii) the restriction $\left.\Phi\right|_{V}$ is $R$-equivariant and preserves the reversible normal linear part $\tilde{X}^{N}=$ $\omega(\nu) \partial_{x}+\Omega(\nu) z \partial_{z}$ of the unperturbed vector field $\tilde{X}$.

Proof. See [Broer et al., 2006] and also in [Ciocci, 2003, Theorem 11, Chapter 7].

\subsection{Persistence of $(n+1)$-tori}

In an open region of parameter space, the surviving $n$-tori are elliptic and can branch off invariant $(n+1)$-tori. This phenomenon is named excitation of normal modes, compare with e.g. [Sevryuk, 1993; Jorba \& Villanueva, 1997; Sevryuk, 1997]. Starting point is the integrable normal form $\tilde{X}$, of which

$$
X=\tilde{X}+\Theta,
$$

is a small perturbation. Our aim is to show persistence of the $(n+1)$-tori, $T_{\nu, \sigma}^{n+1}$, which are determined by the cubic equation (17) in $M$. Here, $\sigma$ is the value of $S$ treated as a distinguished parameter, while $\nu$ is the external parameter, upon which also the coefficients in (17) depend. In the supercritical case $\beta>0$ these tori are elliptic and in the subcritical case we have both hyperbolic and elliptic $(n+1)$-tori that may meet and vanish in a quasiperiodic center-saddle bifurcation during which the tori become parabolic. As $\mu_{2} \rightarrow 0$, the perturbation by both the (integrable) higher order terms of $\tilde{X}$ and the nonintegrable remainder $\Theta$ is sufficiently small to yield persistence of the $(n+1)$-tori by [Broer \& Huitema, 1995] satisfying appropriate Diophantine conditions on the internal frequencies and the normal frequencies.

For normally hyperbolic tori the necessary Diophantine conditions are of the form (5) and only involve the internal frequencies $\left(\omega_{1}, \ldots, \omega_{n}\right)=$ $f(z, \nu)$ with $z$ determined from (17) and

$$
\omega_{n+1}=1+\mu_{1}+\delta \sigma+(\alpha+\gamma) M+\frac{\sigma}{2 M} .
$$

For normally elliptic tori the necessary Diophantine conditions also involve the normal frequency $\omega^{N}$ and read

$$
\left|\langle\omega, k\rangle+k_{n+1} \omega_{n+1}+l \omega^{N}\right| \geq \gamma\left(|k|+\left|k_{n+1}\right|\right)^{-\tau},
$$

for all $\left(k, k_{n+1}\right) \in \mathbb{Z}^{n} \times \mathbb{Z} \backslash\{(0,0)\}$ and $l=0, \pm 1, \pm 2$. Introducing the (conjugated) coordinates

$$
Q=-\frac{1}{2} \ln M \quad \text { and } \quad P=z_{1} z_{3}+z_{2} z_{4},
$$

we have

$$
\omega^{N}=e^{2 Q_{\sigma}} \sqrt{\sigma^{2}+2 \beta e^{-6 Q_{\sigma}}}
$$

with restriction $\sigma^{2} / 2 e^{-6 Q_{\sigma}}>-\beta$ in the subcritical case $\beta<0$.

Theorem 9 (Supercritical case). Let $\tilde{X}$ be the real analytic family of integrable reversible vector fields of Theorem 8. Let $X$ be any real analytic family of reversible vector fields sufficiently close to $\tilde{X}$ in the compact open topology. Assume $\beta>0$ and let $V$ be a neighborhood of $\left(\nu_{0}, \sigma=0\right) \in \mathbb{R}^{q} \times \mathbb{R}$. Then, for $\gamma>0$ sufficiently small and any $(\nu, \sigma) \in V$, there exist a neighborhood $\tilde{V} \subseteq V$ and a map $\Phi: M \times \tilde{V} \rightarrow$ $M \times \mathbb{R}^{q} \times \mathbb{R}$ defined near $T_{\nu, \sigma}^{n+1}$ such that

(i) $\Phi$ is a $C^{\infty}$-near-identity diffeomorphism onto its image;

(ii) the image of Diophantine tori $T_{\nu, \sigma}^{n+1 ; \text { Dioph }}$ under $\Phi$ is a Cantor family of $X$-invariant Diophantine tori and the restriction of $\Phi$ to this family is a conjugacy between $\tilde{X}$ and $X$;

(iii) the restriction $\left.\Phi\right|_{T_{\nu, \sigma}^{n+1 ; \text { Dioph }}}$ is $R$-equivariant and preserves the reversible normal linear part of the unperturbed vector field $\tilde{X}$.

Proof. The result is a direct consequence of [Broer \& Huitema, 1995], see also Sec. 6.2.

Theorem 10 (Subcritical case). Let $\tilde{X}$ be the real analytic family of integrable reversible vector fields of Theorem 8. Let $X$ be any real analytic family of reversible vector fields sufficiently close to $\tilde{X}$ in the compact open topology. Assume $\beta<0$ and let $V$ be a neighborhood of $\left(\nu_{0}, \sigma=0\right) \in \mathbb{R}^{q} \times \mathbb{R}$. Then, for $\gamma>0$ sufficiently small and any $(\nu, \sigma) \in V$,

(i) elliptic case: there exist a neighborhood $\tilde{V}_{\text {ell }} \subseteq V$ and a map $\Phi: M \times \tilde{V}_{\text {ell }} \rightarrow M \times \mathbb{R}^{q} \times \mathbb{R}$ defined near $T_{\nu, \sigma}^{n+1 ; \text { ell }}$ such that a result as in Theorem 9 holds. 
(ii) hyperbolic case: there exist a neighborhood $\tilde{V}_{\text {hyp }} \subseteq V$ and a map $\Phi: M \times \tilde{V}_{\text {hyp }} \rightarrow M \times \mathbb{R}^{q} \times \mathbb{R}$ defined near $T_{\nu, \sigma}^{n+1 \text {;yp }}$ such that a result as before holds, with the parameterizing Cantor set defined by Diophantine conditions involving only the internal frequencies.

Proof. The result follows from [Broer \& Huitema, 1995]. See Sec. 6.2 for more details.

In the integral family $\tilde{X}$, subordinate to the swallowtail singularity one finds reversible center-saddle bifurcations. To our knowledge persistence of this bifurcation has not yet been described completely for the quasi-periodic case. For the Hamiltonian analogue see e.g. [Hanßmann, 1998], where it is conjectured that this theory carries over to the present reversible setting.

\subsection{Persistence of $(n+2)$-tori}

In Sec. 4.1 we claimed the existence of quasiperiodic solutions with two frequencies of the reduced family $\tilde{X}_{\text {red }}$. Their persistence in the case $\beta>0$ has been proven in [Iooss \& Los, 1990]. Regarding the persistence of the corresponding $(n+$ 2)-tori of Corollary 7 we can state the following.

Theorem 11. Let $\tilde{X}$ be the real analytic family of integrable reversible vector fields of Theorem 8. Let $X$ be any real analytic family of reversible vector fields sufficiently close to $\tilde{X}$ in the compact open topology. Let $W$ be a neighborhood of $\left(\nu_{0}, \sigma=0, \eta=\right.$ $0) \in \mathbb{R}^{q} \times \mathbb{R} \times \mathbb{R}$. Then, for $\gamma>0$ sufficiently small and any $(\nu, \sigma, \eta) \in W$, there exist a neighborhood $\tilde{W} \subseteq W$ and a map $\Phi: M \times \tilde{W} \rightarrow M \times \mathbb{R}^{q} \times \mathbb{R} \times \mathbb{R}$ defined near $T_{\nu, \sigma, \eta}^{n+2}$ such that

(i) $\Phi$ is a $C^{\infty}$-near-identity diffeomorphism onto its image;

(ii) the image of Diophantine tori $T_{\nu, \sigma, \eta}^{n+2 ; \text { Dioph }}$ under $\Phi$ is a Cantor family of $X$-invariant Diophantine tori and the restriction of $\Phi$ to this family is a conjugacy between $\tilde{X}$ and $X$;

(iii) the restriction $\left.\Phi\right|_{T_{\nu, \sigma, \eta}^{n+2 ; \text { Dioph }}}$ is $R$-equivariant.

(iv) in the supercritical case $\beta>0$ the nontrivial monodromy of the bundle of $\tilde{X}$-invariant $(n+$ $2)$-tori extends to the nearly-integrable Cantor family $T_{\nu, \sigma, \eta}^{n+2 ; \text { Dioph }}$.

Proof. See Sec. 6.2.

Theorems 8-11 lead to a "Cantorisation" of the local stratifications sketched in Figs. 2 and 3 .
The $q$-dimensional stratum parameterizing invariant $n$-tori, sketched one-dimensional in both figures, turns into the Cantor set sketched in Fig. 5. The $(q+1)$-dimensional stratum parameterizing normally hyperbolic $(n+1)$-tori, sketched as a twodimensional surface in Fig. 3, gets replaced by a Cantor set with $(q-n+1)$-dimensional "arcwise connected components", while the Cantor set replacing the strata parameterizing normally elliptic $(n+1)$-tori have "arcwise connected components" of dimension $q-n$. Finally, the open strata sketched in Figs. 2 and 3 turn into $(q+2)$ dimensional Cantor sets that have $(q-n+1)$ dimensional "arcwise connected components". All these "arcwise connected components" are leafs of foliations that are Whitney- $C^{\infty}$ parameterized over Cantor dust.

\section{Proofs}

In this section we prove the various results of Secs. 4 and 5 .

\subsection{Dynamics of $\tilde{X}_{\text {red }}$}

We now analyze the 4 -D real $R$-reversible vector field $\tilde{X}_{\text {red }}$ and so prove Theorems 3 and 4 . Recall that the system has a 1:1 resonance in the linear part; that is, two pairs of purely imaginary eigenvalues of the linear part collide at $\pm \mathrm{i}\left(1+\mu_{1}\right)$ on the imaginary axis when $\mu_{2}=0$, see Fig. 1 .

\subsubsection{Symmetry reduction}

The vector field (15) has a large symmetry group $\mathbb{T}^{n+1}$ acquired by normalization and furthermore inherited the reversing symmetry $(x, z) \mapsto$ $(-x, R(z))$ from the original system. Reducing the free $\mathbb{T}^{n}$-symmetry $(x, z) \mapsto(x+\xi, z)$ yields the vector field

$$
X_{H}+\alpha Y
$$

whose low order terms are given in (13). Note that this reduces the phase space $\mathcal{M}$ to the Euclidean space $\mathbb{R}^{4}$. The remaining $S^{1}$-symmetry

$$
(x, z) \mapsto\left(x, X_{S}^{t=\rho}(z)\right)
$$

is not free; here $X_{S}^{t=\rho}$ denotes the ( $2 \pi$-periodic) flow of the linear vector field $X_{S}$ at time $\rho$. Indeed, the origin $z=0$ is an equilibrium and correspondingly $X_{S}^{t=\rho}(0)=0$ for all $\rho \in S^{1}=\mathbb{R} / 2 \pi \mathbb{Z}$. Hence, the quotient $\mathcal{P}=\mathbb{R}^{4} / S^{1}$ is not a smooth manifold but 
contains a singular point (from which $z=0$ is reconstructed, from regular points of $\mathcal{P}$ one reconstructs periodic orbits of $X_{S}$ ).

The reduction of the $S^{1}$-symmetry (23) is best performed in terms of the invariants of this group action.

Lemma 12. Let $M, N, S, P$ be the generators of the ring of $S^{1}$-invariant functions given by (14) and (20). Then, the system (13) reduces to the vector field

$$
\begin{aligned}
\dot{M} & =-P \\
\dot{N} & =P q(M, S) \\
\dot{P} & =2 M q(M, S)-2 N \\
\dot{S} & =0
\end{aligned}
$$

with

$$
q(M, S)=\mu_{2}+\beta M+\gamma S .
$$

The phase space is the three-dimensional half-cone

$$
\begin{aligned}
\mathcal{P}:= & \left\{(M, N, P, S) \in \mathbb{R}^{4} \mid 2 M N=\frac{P^{2}+S^{2}}{2},\right. \\
& M \geq 0 \text { and } N \geq 0\}
\end{aligned}
$$

Proof. See [van der Meer, 1985].

Noting that the value $\sigma$ of $S$ is now a (distinguished) parameter for the vector field, we are led to the family of reduced phase spaces

$$
\begin{aligned}
\mathcal{P}_{\sigma}= & \left\{(M, N, P) \in \mathbb{R}^{3} \mid f_{\sigma}(M, N, P)=0,\right. \\
& M \geq 0 \text { and } N \geq 0\}
\end{aligned}
$$

where

$$
f_{\sigma}(M, N, P)=2 M N-\frac{P^{2}+\sigma^{2}}{2} .
$$

Figure 6 depicts the reduced phase space for different values of $\sigma$. The reduced equations of motions are given by $(24 a)-(24 c)$.

Proposition 13. The reduced system (24a)-(24c) is a Poisson system

$$
\begin{gathered}
\dot{M}=\{M, H\}, \quad \dot{N}=\{N, H\} \\
\dot{P}=\{P, H\}
\end{gathered}
$$

with Poisson bracket

$$
\{g, h\}=\left\langle\nabla g \times \nabla h \mid \nabla f_{\sigma}\right\rangle
$$

and Hamiltonian

$H(N, M, P)=N+\mu_{2} M+\gamma S M+\frac{\beta}{2} M^{2}$.

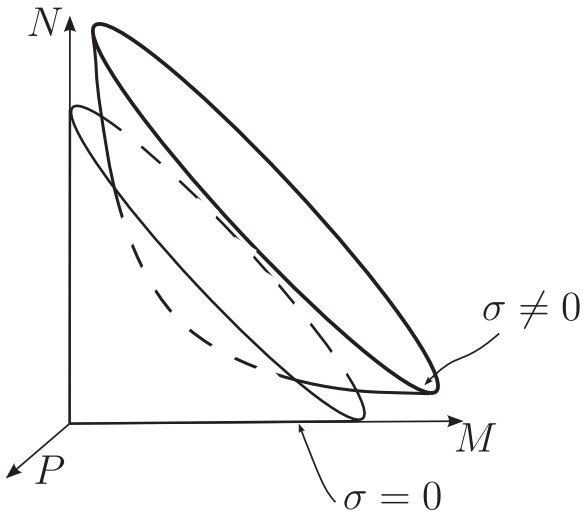

Fig. 6. Level sets of $f_{\sigma}$ for $M, N \geq 0$.

Proof. See [Bridges, 1998, Sec. 2, Theorem 2.1]. Compare also with [van der Meer et al., 1994].

\subsubsection{The reduced dynamics}

Here we briefly describe the 1DOF dynamics defined by the truncated system (27), see also [van der Meer, 1985; Hanßmann \& van der Meer, 2002]. Proposition 13 leads to a geometric characterization of the orbits of (27): the phase curves are obtained intersecting the reduced phase space $\mathcal{P}_{\sigma}$ with the level sets of $H$; see Fig. 7. Transverse intersections yield (relative) periodic orbits, with period related to the area enclosed in $\mathcal{P}_{\sigma}$ by the orbit. The (relative) equilibria correspond to critical points of $H$ on $\mathcal{P}_{\sigma}$, here the energy level set $\{H=h\}$ is tangent to the reduced phase space $\mathcal{P}_{\sigma}$. Since $\{H=h\}$ is a parabolic cylinder and $\mathcal{P}_{\sigma}$ is a surface of revolution this can only happen on the contour $\{P=0\} \cap \mathcal{P}_{\sigma}$. The singular point 0 of $\mathcal{P}_{0}$ is always an equilibrium. Explicit calculations which are given in Appendix B lead to the following result.

Proposition 14. For fixed $\sigma \neq 0$, the equilibria on $\mathcal{P}_{\sigma}$ are determined by the roots of the cubic polynomial

$$
F(M, \sigma):=\sigma^{2}-4 \beta M^{3}-4 \mu_{2} M^{2}-4 \gamma \sigma M^{2},
$$

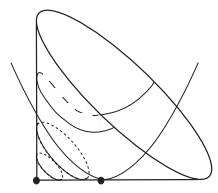

(a)

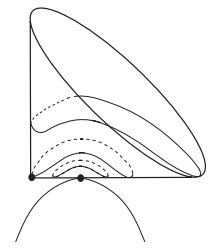

(b)
Fig. 7. Intersections of $\mathcal{P}_{\sigma}$ with the level sets of $H$. (a) Subcritical case $(\beta<0)$, (b) Supercritical case $(\beta>0)$. 
the energy value corresponding to each solution $M$ is

$$
h(M)=\frac{\sigma^{2}}{4 M}+\mu_{2} M+\frac{\beta}{2} M^{2}+\gamma \sigma M .
$$

For fixed $\beta \neq 0$ and $\gamma \in \mathbb{R}$ the solution $M$ of (29) is parameterized by $\left(\mu_{2}, \sigma\right)$ giving part of a swallowtail surface in $\left(\mu_{2}, \sigma, h\right)$-space. For $\sigma=0$, the regular equilibria on $\mathcal{P}_{0}$ are

$$
(M, N, P)=\left(-\frac{\mu_{2}}{\beta}, 0,0\right) .
$$

Proof. See Appendix B.2.

The following proposition summarizes the results about the linear behavior of the equilibria.

Proposition 15. As $\mu_{2}$ passes through zero, a Hamiltonian Hopf bifurcation takes place and we have to distinguish two cases. In the supercritical case $\beta>0$ the $\mu_{2}$-axis $(\sigma, h)=0$ (which corresponds to the singular equilibria) is accompanied by a single sheet $h=h\left(\mu_{2}, \sigma\right)$ corresponding to elliptic regular equilibria that forms a crease at $\left\{\left(\mu_{2}, 0,0\right) \mid \mu_{2}>0\right\}$ where the singular equilibria are elliptic and passes below the thread $\left\{\left(\mu_{2}, 0,0\right) \mid \mu_{2}<0\right\}$ where the singular equilibria are hyperbolic. See also Fig. $7($ a).

In the subcritical case $\beta<0$ there are no regular equilibria for $\mu_{2}<0$. In the wedge

$$
16 \mu_{2}^{3}>27 \beta^{2} \sigma^{2}
$$

there are two sheets $h_{1}\left(\mu_{2}, \sigma\right)<h_{2}\left(\mu_{2}, \sigma\right)$ corresponding to elliptic and hyperbolic regular equilibria, respectively. The lower sheet $h=h_{1}\left(\mu_{2}, \sigma\right)$ forms a crease at $\left\{\left(\mu_{2}, 0,0\right) \mid \mu_{2}>0\right\}$ and meets the (smooth) upper sheet $h=h_{2}\left(\mu_{2}, \sigma\right)$ in a cusp-like fashion with coinciding tangent planes - for $\left(\mu_{2}, \sigma\right)$ in the border

$$
16 \mu_{2}^{3}=27 \beta^{2} \sigma^{2}
$$

of the wedge. See also Fig. $7(b)$.

Proof. See [van der Meer, 1985].

\subsubsection{Effect of conservative higher order terms}

We now consider a reduced normal form (22) that is not already truncated at third order.

Lemma 16. The dynamics of the normalized vector field $X_{H}$ in (22) is after reduction of the $\mathbb{T}^{n}$ symmetry $(x, z) \mapsto(x+\xi, z)$ qualitatively the same as that of system (27) provided that $\beta \neq 0$. In particular, there is the same distinction between the supercritical case $\beta>0$ and the subcritical case $\beta<0$.

Proof. See [van der Meer, 1985, Corollaries 3.373.39].

In the following we shall skip the higher order terms and restrict to the Hamiltonian (28) analyzed in Sec. 6.1.2.

\subsubsection{Reconstruction of the Hamiltonian dynamics in $2 D O F$}

It is straightforward to reconstruct the full dynamics of $X_{H}$ in two degrees of freedom. At regular points of

$$
\mathcal{P}=\bigcup_{\sigma} \mathcal{P}_{\sigma}
$$

we have to superpose a periodic orbit and from the singular point we reconstruct the central equilibria. In the supercritical case almost all orbits of the reduced system are periodic, leading to invariant 2tori in two degrees of freedom. In the subcritical case there are, for $\mu_{2}>0$, some invariant 2-tori as well, but the vast majority of orbits leaves a neighborhood of the origin. The flow is organized by the relative equilibria - from regular equilibria on $\mathcal{P}$ we reconstruct periodic orbits.

Proposition 17. Let $S, M, P$ be as before and define the angle $\theta$ conjugate to $S$ and, locally near periodic orbits, the variable $Q=-(1 / 2) \ln M$ conjugate to $P$ by

$$
\begin{aligned}
& z_{1}=\frac{\sqrt{2} \sin \theta}{\exp Q} \\
& z_{2}=\frac{\sqrt{2} \cos \theta}{\exp Q} \\
& z_{3}=-\frac{S}{\sqrt{2}} \exp Q \cos \theta+\frac{P}{\sqrt{2}} \exp Q \sin \theta \\
& z_{4}=\frac{S}{\sqrt{2}} \exp Q \sin \theta+\frac{P}{\sqrt{2}} \exp Q \cos \theta
\end{aligned}
$$

Then, the vector field $X_{H}$ has the equations of motion

$$
\begin{aligned}
\dot{\theta} & =1+\mu_{1}+\delta S+\gamma \exp (-2 Q)+\frac{S}{2} \exp (2 Q) \\
\dot{S} & =0 \\
\dot{Q} & =\frac{P}{2} \exp (2 Q)
\end{aligned}
$$


$\dot{P}=-\frac{\exp (2 Q)}{2}\left(P^{2}+F(\exp (-2 Q), S)\right)$

with $F$ defined in (29).

Proof. This is a straightforward computation.

In particular, the periodic orbits have the form

$$
\left\{(\theta, S, P, Q) \in S^{1} \times \mathbb{R}^{3} \mid S=\sigma, Q=Q_{\sigma}, P=0\right\}
$$

where $Q_{\sigma}$ is determined by Eq. (17).

\subsubsection{Reconstruction of the 4-D reversible dynamics}

The additional term $\alpha Y$ in (22) is tangent to the fibres of the Hilbert map

$$
\rho: \mathbb{R}^{4} \rightarrow \mathcal{P}, \quad \rho(z)=(M, N, P, S) .
$$

Hence the following holds.

Proposition 18. The dynamics of the vector field $X_{H}+\alpha Y$ as in (22) is determined by the equations of motion (34b)-(34d) as for $X_{H}$ and

$$
\begin{aligned}
\dot{\theta}= & 1+\mu_{1}+\delta S+(\alpha+\gamma) \exp (-2 Q) \\
& +\frac{S}{2} \exp (2 Q) .
\end{aligned}
$$

Proof. See Appendix B.3.

The periodic orbits (35) are left intact but their period is altered. In particular, the Hamiltonian $H$ is invariant under the whole vector field (22), which can also be inferred from $\left[X_{H}, Y\right]=0$.

Remark. This finishes the proof of Theorems 3 and 4.

\subsubsection{Effect of non-Hamiltonian higher order terms}

The tangency of the reversible $\alpha Y$ (cf. (22)) to the fibres of (36) implies that Theorems 3 and 4 remain valid if the truncated vector field (13) is replaced by the reduced normal form $\tilde{X}_{\text {red }}$ of any order.

Proposition 19. Higher order normalization does not alter Eqs. (34b)-(34d), but causes a general additional term $s(\exp (-2 Q), S)$ in $(34 a)$, instead of merely $\alpha \exp (-2 Q)$ in (37).

\section{Proof. See Appendix B.3.}

Hence, higher order normalization does not lead to qualitative changes, provided that we restrict to the supercritical case $\beta>0$ or to the subcritical case $\beta<0$. In this sense the dynamical system defined by (13) is structurally stable. This robustness with respect to normalized higher order terms is inherited by (11) and will be used in what follows to obtain quasi-periodic stability in the sense of [Broer et al., 1990].

\subsection{Proofs of the KAM theorems}

As observed before the persistence of the $n$-tori and $(n+1)$-tori is a direct consequence of respectively [Broer et al., 2006] and [Broer \& Huitema, 1995], while that of the $(n+2)$-tori follows from classical KAM theory, see e.g. [Moser, 1973; Huitema, 1988].

Proof of Theorems 9 and 10. On the phase space $\mathbb{T}^{n} \times \mathbb{R}^{4}=\{x, z\}$ the transformation (34) yields local coordinates around the invariant $(n+1)$-tori $T_{\nu, \sigma}^{n+1}$, which are given by $(S, Q, P)=\left(\sigma, Q_{\sigma}, 0\right)$ where $\sigma \neq$ 0 and $Q_{\sigma}$ is a root of $F(\exp (-2 Q), \sigma)$. Since the right-hand side of

$$
\dot{x}=f\left(z\left(\theta, \sigma, Q_{\sigma}, 0\right), \nu\right)
$$

depends on the $n+1$ st angle $\theta$ one still has to pass to better adapted coordinates on $\mathbb{T}^{n}$, but for our purposes it is not necessary to actually perform that transformation.

The normal linear behavior of $T_{\nu, \sigma}^{n+1}$ is determined by (34c) and (34d), while (34b) confirms that $\sigma$ is an extra parameter (next to $\nu$ ) that the family of $(n+1)$-tori depends upon. Using $F(\exp (-2 Q), \sigma)=0$ in the resulting expression for the normal frequency yields (21) for the elliptic tori. Under the appropriate Diophantine conditions given in Sec. 5.2, the result of [Broer \& Huitema, 1995] applies once it is shown that the perturbation by both the (integrable) higher order terms of $\tilde{X}$ and the nonintegrable remainder $\Theta$ in (19) is sufficiently small. Therefore, we scale $\sigma$ with $\mu_{2}^{3 / 2}$ as the bifurcation parameter $\mu_{2} \rightarrow 0$. To obtain the necessary estimates on the remainder term $\Theta$ in (19) we follow [Broer et al., 2007c] and normalize higher order terms only after passing to the new coordinates provided by Theorem 8 .

Proof of Theorem 11. Persistence of the $(n+2)$-tori is obtained by classical KAM theory, see e.g. [Moser, 1973; Huitema, 1988]. To this end one has to check the required nondegeneracy condition. Here, we use hyperbolicity of lower dimensional tori. In the subcritical case $\beta<0$ this is provided by normally 
hyperbolic $(n+1)$-tori; as these are approached by $(n+2)$-tori, the frequency that we do not directly control by means of the external parameter $\nu$ converges to zero. In the supercritical case $\beta>0$ all $(n+1)$-tori are elliptic, but the hyperbolic $n$-tori form a codimension-four subset in the product of phase space and parameter space that leads to non-trivial monodromy, cf. [Efstathiou, 2005; Broer et al., 2007c; Hoo, 2005]. Again this yields the necessary variation of the "uncontrolled" frequencies.

By the methods of Broer et al. [2007a] one can furthermore glue the resulting local KAMconjugacies together to a globally Whitney-smooth conjugacy from the integrable to the near-integrable Cantor family of tori.

\section{Further Remarks}

(i) In a more general setting the phase space is $M=\mathbb{T}^{n} \times \mathbb{R}^{m} \times \mathbb{R}^{2 p}=\{x, y, z\}$, with $m \geq 0$ and $p \geq 2$. The reversor $G$ reads $G(x, y, z)=$ $(-x, y, R z)$ with $R$ as before. In this more general situation we say that $G$ is of type $(n+p$, $m+p)$, noting that $G$ then has an $(m+p)$ dimensional submanifold of fixed points. The present considerations are for the case where $G$ is of type $(n+2,2)$.

In case we have $y$-variables as well, the corresponding part $g \partial / \partial y$ in the vector field has to satisfy $g(-x, y, R z) \equiv-g(x, y, z)$ from which $g(y, 0)=0$ for $x$-independent $g$ immediately follows. While linear terms in $z$ as in [Wei, 2001] may still occur, the scaling given in [Broer \& Huitema, 1995; Ciocci, 2003; Broer et al., 2006] makes these of order $\varepsilon$. In this way the unperturbed normal linear part again gets the desired form (4), where we have acquired $m$ additional, distinguished parameters related to the $y$-variables, that can help to effectuate the BHT nondegeneracy condition. In particular, if $m \geq n+2$ there is no need for external parameters $\nu$ at all.

(ii) It is instructive to compare the quasi-periodic reversible Hopf bifurcation with the Hamiltonian case. The reduced integrable systems on $\mathbb{R}^{4}$ define the same geometry, although the dynamics in the reversible case is slightly different due to the additional drift $\alpha \exp (-2 Q)$ in (37) along the $S^{1}$-fibres. In particular, for the supercritical cases $\beta>0$ we have monodromy both in the Hamiltonian and the reversible setting.

For a Hamiltonian system the full phase space has to provide actions conjugate to the toral angles on $\mathbb{T}^{n}$, leading to $\mathbb{T}^{n} \times \mathbb{R}^{n} \times$ $\mathbb{R}^{4}$ which corresponds to a reversor of type $(n+2, n+2)$ in the reversible context. For Hamiltonian as for reversible systems it would be interesting to consider $p$-fold resonances as in [Knobloch \& Vanderbauwhede, 1995, 1996] under quasi-periodic forcing. Note that Theorem 8 admits a straightforward generalization to the case $p \geq 3$.

\section{Acknowledgments}

We thank Gerard Iooss, André Vanderbauwhede and the referee for helpful comments. M. C. Ciocci acknowledges support by the European Community's 6th Framework Programme, Marie Curie IntraEuropean Fellowship EC contract Ref. MEIFCT-2005-515291, award Nr. MATH-P00286.

\section{References}

Arnold, V. I. [1971] "On matrices depending on parameters," Russ. Math. Surv. 26, 29-43.

Braaksma, B. L. J. \& Broer, H. W. [1987] "On a quasiperiodic Hopf bifurcation," Ann. Inst. Henri Poincaré 4, 115-168.

Bridges, T. [1998] "Poisson structure of the reversible 1:1 resonance," Physica D 112, 40-49.

Broer, H. W., Huitema, G. B., Takens, F. \& Braaksma, B. L. J. [1990] "Unfoldings and bifurcations of quasiperiodic tori," Mem. Amer. Math. Soc. 83, 1-175.

Broer, H. W. \& Vegter, G. [1992] "Bifurcational aspects of parametric resonance," Dyn. Rep., New Series 1, $1-51$.

Broer, H. W. [1993] "Notes on perturbation theory 1991," Erasmus ICP Mathematics and Fundamental Applications (Aristotle University, Thessaloniki).

Broer, H. W. \& Huitema, G. B. [1995] "Unfoldings of quasi-periodic tori in reversible systems," J. Dyn. Diff. Eq. 7, 191-212.

Broer, H. W., Huitema, G. B. \& Sevryuk, M. B. [1996] Quasi-Periodic Motions in Families of Dynamical Systems: Order Amidst Chaos, Lecture Notes in Mathematic, Vol. 1645 (Springer, Berlin).

Broer, H. W., Cushman, R. H., Fassò, F. \& Takens, F. [2007a] "Geometry of KAM-tori for nearly integrable Hamiltonian systems," Erg. Th. Dyn. Syst. 27 $725-741$. 
Broer, H. W., Hoo, J. \& Naudot, V. [2007b] "Normal linear stability of quasi-periodic tori," J. Diff. Eq. 232, $355-418$.

Broer, H. W., Hanßmann, H. \& Hoo, J. [2007c] "The quasi-periodic Hamiltonian Hopf bifurcation," Nonlinearity 20, 417-460.

Broer, H. W., Ciocci, M. C. \& Vanderbauwhede, A. [2006] "Normal 1:1 resonance of invariant tori in reversible systems: A study in quasi-periodic bifurcation theory," (in preparation).

Ciocci, M. C. [2003] Bifurcations of Periodic Points in Families of Reversible Mappings and of QuasiPeriodic Solutions in Reversible Systems, $\mathrm{PhD}$ thesis (Ghent University, Belgium). Available online at: http://www.lib.ugent.be/execl/infod/ethesis.shtml (accessed 28 February 2006).

Ciocci, M. C. [2005] "Persistence of quasi-periodic orbits in families of reversible systems with a 1:1 resonance," Proc. Equadiff 03, Hasselt 2003, eds. Dumortier, F. et al. (World Scientific, Singapore), pp. 720-725.

Cushman, R. H. \& Sanders, J. A. [1986] "Nilpotent normal forms and representation theory of $\operatorname{sl}(2, \mathbb{R}), "$ Multiparameter Bifurcation Theory (Arcata, 1985), Contemp. Math. 56, 31-51.

Efstathiou, K., Cushman, R. H. \& Sadovskií, D. A. [2004] "Hamiltonian Hopf bifurcation of hydrogen in crossed fields," Physica D 194, 250-274.

Efstathiou, K. [2005] Metamorphoses of Hamiltonian Systems with Symmetries, Lecture Notes in Mathematics, Vol. 1864 (Springer, Berlin).

Gibson, C. G. [1979] Singular Points of Smooth Mappings (Pitman, London).

Hanßmann, H. [1998] "The quasi-periodic centre-saddle bifurcation," J. Diff. Eq. 142, 305-370.

Hanßmann, H. \& Meer, J. C. van der [2002] "On the Hamiltonian Hopf bifurcations in the $3 D$ HénonHeiles family," J. Dyn. Diff. Eq. 14, 675-695.

Hirsch, M. W. \& Smale, S. [1974] Differential Equations, Dynamical Systems, and Linear Algebra (Academic Press, NY).

Hoo, J. [2005] Quasi-Periodic Bifurcations in a Strong Resonance, $\mathrm{PhD}$ thesis (Rijksuniversiteit Groningen, The Netherlands).

Huitema, G. B. [1988] Unfoldings of Quasi-Periodic Tori, $\mathrm{PhD}$ thesis (Rijksuniversiteit Groningen, The Netherlands).

Iooss, G., Mielke, A. \& Demay, Y. [1989] "Theory of steady Ginzburg-Landau equation in hydrodynamic stability problems," European J. Mech. B/Fluids 8, 229-268.

Iooss, G. \& Los, J. [1990] "Bifurcation of spatially quasiperiodic solutions in hydrodynamic stability problems," Nonlinearity 3, 851-871.

Jorba, À. \& Villanueva, J. [1997] "On the normal behaviour of partially elliptic lower-dimensional tori of Hamiltonian systems," Nonlinearity 10, 783-822.
Knobloch, J. \& Vanderbauwhede, A. [1995] "Hopf bifurcation at a $k$-fold resonances in reversible systems," preprint N. M16/95 (Technische Universität Ilmenau).

Knobloch, J. \& Vanderbauwhede, A. [1996] "Hopf bifurcation at $k$-fold resonances in conservative systems," Nonlinear Dynamical Systems and Chaos, Groningen 1995, eds. Broer, H. W. et al., Progress in Nonlinear Differential Equations and Their Applications, Vol. 19 (Birkhäuser, Basel), pp. 155-170.

Lamb, J. S. W. [1994] Reversing Symmetries in Dynamical Systems, $\mathrm{PhD}$ thesis (Universiteit van Amsterdam, The Netherlands).

Lamb, J. S. W., Teixeira, M. A. \& Yang, J. [2001] "On the Hamiltonian structure of normal forms for elliptic equilibria of reversible vector fields in $\mathbb{R}^{4}$," preprint (Imperial College London).

Mielke, A. [1986] "A reduction principle for nonautonomous systems in infinite dimensional spaces," J. Diff. Eq. 65, 68-88.

Moser, J. [1973] Stable and Random Motions in Dynamical Systems (Princeton University Press, Princeton).

Rink, B. [2002] "Direction-reversing traveling waves in the even Fermi-Pasta-Ulam lattice," J. Nonlin. Sci. 12, 479-504.

Sevryuk, M. B. [1986] Reversible Systems, Lecture Notes in Mathematics, Vol. 1211 (Springer, Berlin).

Sevryuk, M. B. [1993] "Invariant tori of reversible systems of intermediate dimensions," Russ. Akad. Sci. Dokl. Math. 47, 129-133.

Sevryuk, M. B. [1997] "Invariant tori of intermediate dimensions in Hamiltonian systems," Reg. Chaot. Dyn. 2, 30-40.

van der Meer, J. C. [1985] The Hamiltonian Hopf Bifurcation, Lecture Notes in Mathematics, Vol. 1160 (Springer, Berlin).

van der Meer, J. C., Sanders, J. A. \& Vanderbauwhede, A. [1994] "Hamiltonian structure of the reversible nonsemisimple 1:1 resonance," Dynamics, Bifurcation and Symmetry, Cargése 1993, ed. Chossat, P. (Kluwer, Dordrecht), pp. 221-240.

Vanderbauwhede, A. [1990] "Hopf bifurcation for equivariant conservative and time-reversible systems," Proc. Roy. Soc. Edinburgh A 116, 103-128.

Wei, B. [2001] "Perturbations of lower dimensional tori in the resonant zone for reversible systems," JMAA 253, 558-577.

\section{Appendix A}

\section{Reversible Normal Form Theory}

First we develop normal forms of integrable reversible vector fields, later on discussing the near-integrable case. We largely follow [Broer, 1993; Broer \& Huitema, 1995]. 


\section{A.1. Nonlinear considerations in the integrable case}

We begin with integrable reversible systems, i.e. which are invariant under the natural $\mathbb{T}^{n}$-action.

1. Reduction of the $\mathbb{T}^{n}$-symmetry leads from (2) to a reduced family

$$
X_{\text {red }}(z, \nu)=h(z, \nu) \frac{\partial}{\partial z},
$$

of vector fields on $\mathbb{R}^{4}$, where the interest is with (relative) equilibria, which now are involved in a standard reversible Hopf bifurcation, cf. [Bridges, 1998; van der Meer et al., 1994], see also Sec. 6.1.

2. This study admits also a standard normal form approach, which fits in the general Lie-algebra approach. Indeed, by $R$-equivariant transformations the $R$-reversible character is preserved, cf. [Broer \& Huitema, 1995]. Now by such equivariant transformations, infinitesimally generated by equivariant vector fields, the Taylor series of the reduced family $X_{\text {red }}$ can be simplified in the following sense.

For simplicity we assume that the reversible Hopf bifurcation takes place at $\nu=0$, we consider the linear part

$$
\Omega_{0}=S_{0}+N_{0},
$$

canonically split in semisimple and nilpotent part. Accordingly, we consider the linear vector field

$$
A_{\text {red }}=\Omega_{0} \frac{\partial}{\partial z}=A_{S}+A_{N}
$$

which again is the canonical splitting. Normalization formally takes place on the gradation

$$
\prod_{j=1}^{\infty} \mathcal{H}_{\mathrm{red},-R}^{j}
$$

of the space of formal series, where $\mathcal{H}_{\text {red, }-R}^{j}$ contains all polynomial vector fields on $\mathbb{R}^{4}$, the coefficient functions of which are homogeneous polynomials in $(z, \nu)$ of degree $j$. The elements of $\mathcal{H}_{\text {red, }-R}^{j}$ are all $R$-reversible, compare with (3). We recall that here we have to consider the adjoint operators

$$
\operatorname{ad}^{j} A_{\text {red }}: \mathcal{H}_{\text {red, }+R}^{j} \rightarrow \mathcal{H}_{\text {red,-R }}^{j},
$$

defined by Lie-brackets. Here, by induction with respect to the degree $j \in \mathbb{N}$, all terms in the corresponding image im ad ${ }^{j} A_{\text {red }} \subseteq \mathcal{H}_{\text {red,-R }}^{j}$ can be taken out, thereby only leaving terms in a suitable complementary space $\mathrm{C}^{j} \subseteq \operatorname{ker} \operatorname{ad}^{j} A_{S} \subseteq$ $\mathcal{H}_{\text {red, }, R}^{j}$.

The fact that $\mathrm{C}^{j} \subseteq \operatorname{kerad}^{j} A_{S}$ implies $S^{1}$ symmetry of the normal form. For the precise choice of the $\mathrm{C}^{j}, j \in \mathbb{N}$, such that

$$
\operatorname{im~ad}^{j} A_{\text {red }} \oplus C^{j}=\mathcal{H}_{\text {red, }-R}^{j}
$$

as a direct sum splitting, there exist several procedures. In the present paper we follow the $\mathfrak{s l}(2, \mathbb{R})$-approach described in [Cushman \& Sanders, 1986].

\section{A.2. Application to the 1:1 resonance}

We now turn to the case of our particular interest, namely the reversible 1:1-resonance of Sec. 4.1.

The reversor on $\mathbb{R}^{4}=\left\{z_{1}, z_{2}, z_{3}, z_{4}\right\}$ is given by

$$
R:\left(z_{1}, z_{2}, z_{3}, z_{4}\right) \mapsto\left(z_{1},-z_{2},-z_{3}, z_{4}\right),
$$

compare with Proposition 1.

The linear part on the reduced phase space $\mathbb{R}^{4}$ reads $A_{S}+A_{N}$, where

$$
A_{S}=z_{2} \frac{\partial}{\partial z_{1}}-z_{1} \frac{\partial}{\partial z_{2}}+z_{4} \frac{\partial}{\partial z_{3}}-z_{3} \frac{\partial}{\partial z_{4}},
$$

which generates a diagonal action of $S^{1}=S O(2)$ on $\mathbb{R}^{4}$. Observe that the $R$-equivariant functions with the $S O(2)$-symmetry even have an $O(2)$-symmetry.

The invariant functions with respect to the $O(2)$-action form a ring generated by the Hilbert basis (14). This allows to write down the reversible normal form with respect to $A_{S}$ as a sum

$$
r \cdot A_{N}+q \cdot A_{M}+p \cdot A_{S}
$$

where

$$
A_{M}=z_{1} \frac{\partial}{\partial z_{3}}+z_{2} \frac{\partial}{\partial z_{4}}
$$

and where the coefficient functions $r, q$ and $p$ are invariant, i.e. depend on $z$ only as functions of the basic polynomials $M, S$ and $N$.

\section{Normalizing with respect to the nilpotent part}

$$
A_{N}=-z_{3} \frac{\partial}{\partial z_{1}}-z_{4} \frac{\partial}{\partial z_{2}}
$$

of the linear part $A_{S}+A_{N}$ follows [Cushman \& Sanders, 1986]. Here we may achieve $r \equiv 1$ and 
make $p$ and $q$ independent of $N$ whence the normal form in the $z$-direction reads

$$
\omega^{N} A_{S}+A_{N}+q(M, S) A_{M}+p(M, S) A_{S},
$$

with $\omega^{N}+p(0,0)=1+\mu_{1}$ and $q(0,0)=\mu_{2}$.

\section{A.3. Quasi-periodic normal forms}

We conclude with nonlinear considerations in the nonintegrable case, adapting the above normalization procedure as follows, cf. [Braaksma \& Broer, 1987; Broer et al., 1990; Broer, 1993; Broer \& Vegter, 1992].

1. We now consider formal vector fields

$$
Y=F(x, z, \nu) \frac{\partial}{\partial x}+H(x, z, \nu) \frac{\partial}{\partial z},
$$

where $F$ and $H$ are formal series in $(z, \nu)$ with coefficients that are real analytic functions (i.e. convergent) in $x$. This space can be graded to

$$
\prod_{j=1}^{\infty} \mathcal{H}_{-R}^{j}
$$

as before, where we need that

$$
Y \in \mathcal{H}_{-R}^{j} \Leftrightarrow \operatorname{deg} F=j-1, \operatorname{deg} H=j,
$$

and the projections of a reversible vector field to each $\mathcal{H}_{-R}^{j}$ again are reversible in the sense of (3). The degree again refers to the variables $(z, \nu)$. Note that the sets $\mathcal{H}_{-R}^{j}$ so become infinite dimensional vector spaces.

2. We replace the above linear part $A_{\text {red }} \in \mathcal{H}_{\text {red,-R }}^{1}$ by $A \in \mathcal{H}_{-R}^{1}$, defined as

$$
A=\omega \frac{\partial}{\partial x}+A_{\text {red }}=\omega \frac{\partial}{\partial x}+\Omega \frac{\partial}{\partial z} .
$$

3 . The rest of the normalization procedure largely works as before. Indeed, we split into canonical form

$$
A=\omega \frac{\partial}{\partial x}+A_{\text {red }}=\omega \frac{\partial}{\partial x}+A_{S}+A_{N},
$$

where now

$$
\omega \frac{\partial}{\partial x}+A_{S}
$$

is the semi-simple part.

4. As before all terms in the image $\operatorname{imad}^{j} \subseteq$ $\mathcal{H}_{-R}^{j}$ can be taken away by equivariant transformation, infinitesimally generated by equivariant vector fields $Y \in \mathcal{H}_{R}^{j}$.

The construction of the complementary spaces $\mathrm{C}^{j}, j \in \mathbb{N}$, can be done exactly as in the integrable case. This is essentially a finite dimensional problem.

\section{Appendix B}

\section{Hamiltonian Aspects}

Here we focus on the Hamiltonian aspects of our problem (see also e.g. [van der Meer et al., 1994; Lamb et al., 2001; Broer et al., 2007c; Hoo, 2005]) and prove various statements about the integrable normal form.

\section{B.1. Proof of Proposition 2}

The vector field (A.2) can be expressed as the sum of a vector field pointing in the $A_{S}$-direction and a Hamiltonian vector field with respect to the symplectic structure $\mathrm{d} z_{4} \wedge \mathrm{d} z_{2}+\mathrm{d} z_{3} \wedge \mathrm{d} z_{1}$ (which is again invariant under the action of our symmetry group). In this case the linear vector fields $A_{S}, A_{N}, A_{M}$ are the Hamiltonian vector fields to quadratic Hamiltonian functions $S, N, M$ :

$$
A_{S}=X_{S}, \quad A_{N}=X_{N}, \quad A_{M}=X_{M} .
$$

In particular, we may write the normalized vector field as

$$
\omega \frac{\partial}{\partial x}+X_{H}+\alpha Y
$$

with a Hamiltonian function

$$
\begin{aligned}
H(S, N, M)= & \omega^{N} S+N+\lambda M+\frac{\beta}{2} M^{2} \\
& +\gamma S M+\frac{\delta}{2} S^{2}+\text { h.o.t. }
\end{aligned}
$$

and a vector field $Y$ that is parallel to $X_{S}$ and vanishes at $\{M=0\}$. Where the nondegeneracy condition $\beta \neq 0$ is satisfied we may discard the higher order terms and similarly restrict to $Y=M X_{S}$. Since we have dependence on external parameters $\mu$ all "greek" coefficients are functions of $\mu$ with in particular $\omega^{N}(\mu)=1+\mu_{1}$ and $\lambda(\mu)=\mu_{2}$ while the nondegeneracy condition reads $\beta(0) \neq 0$ (whence we restrict to a small neighborhood of $\mu=0$ where $\beta(\mu) \neq 0$ for all $\mu$ ).

Remark.

1. In [Bridges, 1998; van der Meer et al., 1994] it is proven that a $4-\mathrm{D}$ reversible vector field at a $1: 1$ resonance can be seen as a non-Hamiltonian perturbation, of at least order two, of a Hamiltonian vector field. The non-Hamiltonian trajectories 
coincide with the orbits of the vector field associated to the $S^{1}$-action of the semisimple $S_{0}$ and do therefore vanish on the reduced phase space. The phase space of (13) can be fibered into invariant surfaces given by $S=$ const and $H=$ const. In contrast with a "purely" Hamiltonian case, the flow on such fibres is not Hamiltonian since there is a shift along the trajectories of $S_{0}$.

2. We may in fact work with $Y=S X_{M}$ or use any linear combination of these two for $Y$ (except for the Hamiltonian linear combination $S X_{M}+M X_{S}=X_{M S}$ of course), but the previous remark clearly shows that the above choice is the best.

3. In [Lamb et al., 2001] it is shown that a 4-D reversible vector field $\tilde{X}$ in truncated normal form, with 1:1 resonance, is orbitally equivalent to an integrable Hamiltonian vector field $\widehat{X}$. That is, there exists a nonzero function $\varphi$ and a change of coordinates bringing $\varphi \cdot \tilde{X}$ to $\widehat{X}$. In our context, this means that $\alpha=0$ can always be achieved if nontrivial time-scalings are allowed.

\section{B.2. The reduced dynamics}

Here we give the details which prove Proposition 14.

The 1DOF dynamics defined by (27) have been described in [van der Meer, 1985; Hanßmann \& van der Meer, 2002], so we can be brief here. Indeed, the phase curves can be obtained intersecting the reduced phase space $\mathcal{P}_{\sigma}$ with the level sets of (28)

$$
\begin{gathered}
\left\{(M, N, P) \in \mathcal{P}_{\sigma} \mid H(M, N, P)=h\right\} \\
=\mathcal{P}_{\sigma} \cap\{H=h\} \subseteq \mathbb{R}^{3} .
\end{gathered}
$$

Remark. The ultimate reason for all equilibria of (27) on $\mathcal{P}_{\sigma}$ satisfying $\{P=0\}$ is that the reduced system is reversible with respect to

$$
R:(M, N, P) \mapsto(M, N,-P) .
$$

Note that we refrain from reducing the corresponding symmetry group $\mathbb{Z}_{2}$. Indeed, on

$$
\begin{aligned}
\mathcal{P}_{\sigma / \mathbb{Z}_{2}}= & \left\{(M, N) \in \mathbb{R}^{2} \mid M \geq 0, N \geq 0\right. \\
& \left.2 M N \geq \frac{1}{2} \sigma^{2}\right\}
\end{aligned}
$$

it is not possible to define reduced dynamics. Thus, a periodic orbit on $\mathcal{P}_{\sigma}$ is mapped to the part of the parabola

$$
N=h-\mu_{2} M-\frac{\beta}{2} M^{2}-\gamma \sigma M
$$

that lies within the region (B.2) of $\mathbb{R}^{2}$. The two endpoints correspond to the turning points where $\dot{M}=\dot{N}=0$ (since $P=0$ ) and $\dot{P}>0$ or $\dot{P}<0$, respectively. For examples where the passage to the "fully reduced phase space" (B.2) is in fact helpful as it lowers the degrees of certain polynomials generalizing (B.3) see [Rink, 2002; Efstathiou et al., 2004].

To compute the equilibria on $\mathcal{P}_{\sigma}, \sigma \neq 0$ fixed, we determine the points where the parabola (B.3) touches the hyperbola

$$
N=\frac{\sigma^{2}}{4 M}
$$

Since the value $h$ of the energy can always be adjusted appropriately this amounts to equating the derivatives of (B.3) and (B.4) and leads to the cubic equation

$$
F(\sigma, M)=0,
$$

with $F(\sigma, M)$ defined in $(29)$.

For a solution $M$ of (B.5) the appropriate energy value is (30). When $\sigma=0$ we similarly obtain the regular equilibria (31) on $\mathcal{P}_{0}$. Fixing $\beta \neq$ 0 and $\gamma \in \mathbb{R}$ the solution $M$ of (B.5) is parameterized by $\left(\mu_{2}, \sigma\right)$ and we obtain part of a swallowtail surface in $\left(\mu_{2}, \sigma, h\right)$-space, see [van der Meer, 1985; Hanßmann \& van der Meer, 2002] for more details. The linear behavior of the regular equilibria is determined by comparing the curvatures of the parabola (B.3) and the hyperbola (B.4). Indeed, where

$$
\frac{\sigma^{2}}{8 M^{3}}>-\beta
$$

the surface of revolution $\mathcal{P}_{\sigma}$ lies completely above the parabolic cylinder $\{H=h\}$ and the equilibrium $\mathcal{P}_{\sigma} \cap\{H=h\}$ is elliptic. In the supercritical case we have $-\beta<0$ and this holds true for all regular equilibria. In the subcritical case only the equilibria corresponding to the lower sheet $h=h_{1}\left(\mu_{2}, \sigma\right)$ are elliptic. An equilibrium $(M, N, 0)$ accounted for on the upper sheet $h=h_{2}\left(\mu_{2}, \sigma\right)$ satisfies

$$
\frac{\sigma^{2}}{8 M^{3}}<-\beta
$$

whence $\mathcal{P}_{\sigma}$ and $\{H=h\}$ intersect along separatrices and the equilibrium is hyperbolic. When crossing the line (32) a (subordinate) center-saddle bifurcation takes place as an elliptic equilibrium accounted for on the lower sheet meets a hyperbolic equilibrium accounted for on the upper sheet 
and both vanish. In particular, the equilibria corresponding to $h_{1}\left(\mu_{2}, \sigma\right)=h_{2}\left(\mu_{2}, \sigma\right)$, with $\left(\mu_{2}, \sigma\right)$ satisfying (32), are parabolic as (B.3) and (B.4) have third order contact. There is always quadratic contact in the $P$-direction.

\section{B.3. Effect of conservative higher order terms}

We prove Propositions 18 and 19.

We now consider a normal form that is not already truncated at third order. Reducing the free $\mathbb{T}^{n}$-symmetry $(x, z) \mapsto(x+\xi, z)$ yields again a fourdimensional vector field of the form (22), but now with a more general Hamiltonian

$$
H(z)=S(z)+N(z)+r(M(z), S(z))
$$

where the function $r: \mathbb{R}^{2} \rightarrow \mathbb{R}^{2}$ satisfies

$$
\begin{gathered}
\frac{\partial r}{\partial M}(0,0)=\mu_{2}, \quad \frac{\partial r}{\partial S}(0,0)=\mu_{1}, \quad r(0,0)=0, \\
\frac{\partial^{2} r}{\partial M^{2}}(0,0)=\beta, \quad \frac{\partial^{2} r}{\partial M \partial S}(0,0)=\gamma, \\
\frac{\partial^{2} r}{\partial S^{2}}(0,0)=\delta .
\end{gathered}
$$

The vector field $Y$ is parallel to $X_{S}$ and vanishes at $\{M=0\}$, more precisely we have

$$
\alpha Y=s(M, S) X_{S}
$$

with

$$
s(0, S)=0 \forall_{S} \quad \text { and } \quad \frac{\partial s}{\partial M}(0,0)=\alpha .
$$

The functions $r$ and $s$ are related to $p$ and $q$ in (A.2) by

$$
\begin{aligned}
& p(M, S)=\frac{\partial r}{\partial S}(M, S)+s(M, S) \\
& q(M, S)=\frac{\partial r}{\partial M}(M, S) .
\end{aligned}
$$

Since $Y$ remains tangent to the fibres of the Hilbert map (36) the reduced 1DOF system has equations of motion (27) with Hamiltonian

$$
H=N+\hat{r}(M, \sigma)=N+\int_{0}^{M} q_{\sigma}(m) \mathrm{d} m
$$

where $q_{\sigma}(M)=q(M, \sigma)$ and $\hat{r}(M, \sigma)=r(M, \sigma)-$ $r(0, \sigma)$, which amounts to skipping the constant terms $\sigma+r(0, \sigma)$.

To adapt the formulas we obtained in Sec. 6.1.2 to the present dynamics we have to replace the parabola (B.3) by

$$
N=h-\hat{r}(M, \sigma)
$$

whence (29) turns into

$$
\hat{F}(M, \sigma)=\sigma^{2}-4 M^{2} \frac{\partial \hat{r}}{\partial M}(M, \sigma) .
$$

We see that there are no qualitative differences under the condition $\beta \neq 0$; both in the supercritical case $\beta>0$ and in the subcritical case $\beta<0$ the description in Sec. 6.1.2 remains valid and only the exact location of the (relative) equilibria is slightly shifted. 